\title{
Application of effective field theory to finite-volume effects in $a_{\mu}^{\mathrm{HVP}}$
}

\author{
Christopher Aubin $\odot,{ }^{1}$ Thomas Blum, ${ }^{2}$ Maarten Golterman $\odot,{ }^{3}$ and Santiago Peris $\oplus^{4}$ \\ ${ }^{1}$ Department of Physics and Engineering Physics, Fordham University, \\ Bronx, New York, New York 10458, USA \\ ${ }^{2}$ Physics Department, University of Connecticut, Storrs, Connecticut 06269, USA \\ ${ }^{3}$ Department of Physics and Astronomy, San Francisco State University, \\ San Francisco, California 94132, USA \\ ${ }^{4}$ Department of Physics and BIST, Universitat Autònoma de Barcelona, \\ E-08193 Bellaterra, Barcelona, Spain
}

(Received 23 August 2020; accepted 20 October 2020; published 16 November 2020)

\begin{abstract}
One of the more important systematic effects affecting lattice computations of the hadronic vacuum polarization contribution to the anomalous magnetic moment of the muon, $a_{\mu}^{\mathrm{HVP}}$, is the distortion due to a finite spatial volume. In order to reach subpercent precision, these effects need to be reliably estimated and corrected for, and one of the methods that has been employed for doing this is finite-volume chiral perturbation theory. In this paper, we argue that finite-volume corrections to $a_{\mu}^{\mathrm{HVP}}$ can, in principle, be calculated at any given order in chiral perturbation theory. More precisely, once all low-energy constants needed to define the effective field theory representation of $a_{\mu}^{\mathrm{HVP}}$ in infinite volume are known to a given order, also the finite-volume corrections can be predicted to that order in the chiral expansion.
\end{abstract}

DOI: 10.1103/PhysRevD.102.094511

\section{INTRODUCTION}

Recent years have seen renewed efforts to obtain a more reliable and more precise Standard-Model estimate of the muon anomalous magnetic moment. The discrepancy between the best Standard-Model estimates and the experimental value from the Brookhaven experiment [1] has not only persisted, but also has become more acute, as many of the systematic errors associated with the Standard-Model estimate have become more controlled. According to a recent review of the Standard-Model calculation [2] the best average Standard-Model value is estimated to be $3.7 \sigma$ smaller than the value reported in Ref. [1].

The renewed efforts to improve the Standard-Model estimate are driven by new experimental programs at both Fermilab [3] and J-PARC [4], which aim to improve on the precision of the measurement of the magnetic moment, in the case of Fermilab, by a factor of 4. It is thus important to improve the precision of the Standard-Model estimate to a level commensurate with the experimental goal.

The bulk of the error of the Standard-Model estimate originates from the hadronic contributions to the anomalous magnetic moment, which cannot be computed in

Published by the American Physical Society under the terms of the Creative Commons Attribution 4.0 International license. Further distribution of this work must maintain attribution to the author(s) and the published article's title, journal citation, and DOI. Funded by SCOAP ${ }^{3}$. perturbation theory. The hadronic contribution consists of two parts: the hadronic vacuum polarization (HVP) contribution and the hadronic light-by-light contribution; in this paper, the focus will be the HVP contribution. The most precise estimate has been based on the dispersive, data-driven approach, but more recently lattice QCD computations have started to become more precise and are expected to become competitive in the near future. It is thus important to gain a thorough understanding of the various systematic errors afflicting lattice computations of the HVP contribution to the muon anomalous magnetic moment, $a_{\mu}^{\mathrm{HVP}}$.

Lattice computations of the HVP are necessarily done in a finite physical spatial volume, typically a cubic volume with periodic boundary conditions. ${ }^{1}$ As $a_{\mu}^{\mathrm{HVP}}$ is dominated by momenta at a scale set by the muon mass, which is roughly equal to the pion mass, it turns out that finitevolume (FV) effects constitute one of the more important systematic errors in state-of-the-art lattice computations of the HVP. These effects are large enough that lattice results for $a_{\mu}^{\mathrm{HVP}}$ need to be corrected, and it is thus important to compute this correction, as well as the systematic errors associated with such a correction, reliably. Various methods have been used to provide reliable estimates: an adaptation

\footnotetext{
${ }^{1}$ The Euclidean time extent of the lattice is usually significantly larger than the linear spatial dimension. We will not consider effects of the finite extent of the lattice in Euclidean time in this paper.
} 
[5] of the Gounaris-Sakurai model [6] for the low-momentum HVP to finite volume using the method of Ref. [7]; chiral perturbation theory (ChPT) [8-11] in finite volume [12]; a model for low-energy pions including the $\rho$ and $\omega$ resonances [13]; a systematic estimate of the leading FV effects in terms of the forward Compton amplitude of the pion [14,15], based on the methods of Ref. [16]; and, finally, by varying the lattice volume directly in the numerical computations of $a_{\mu}^{\mathrm{HVP}}[11,17]$.

Because the relevant scale is so low, the proper systematic effective field theory (EFT) to analyze these FV effects is ChPT. In Ref. [10], we computed FV corrections to next-tonext-to-leading order (NNLO), i.e., to two loops in ChPT. ${ }^{2}$ Since the lowest-order pionic contribution to $a_{\mu}^{\mathrm{HVP}}$ already involves a pion loop, we will follow the convention of referring to the lowest-order contribution as NLO, the contribution that involves two loops in ChPT as NNLO, etc.

Our motivation here is not to push this to higher orders but to consider whether, as a matter of principle, $\mathrm{FV}$ corrections for $a_{\mu}^{\mathrm{HVP}}$ can be computed to arbitrary orders in ChPT. Even if orders beyond NNLO may never be pursued in practice, it is important to establish that ChPT allows, in principle, a systematic approach to FV effects which is well defined at each order in the chiral expansion. Our main motivation is to illustrate how the properties of an EFT guarantee this to happen through simple examples.

The HVP contribution to the muon anomalous magnetic moment to lowest order in $\alpha$ is given by [18] (see also Ref. [19])

$$
a_{\mu}^{\mathrm{HVP}}=4 \alpha^{2} \int_{0}^{\infty} d q^{2} f\left(q^{2}\right) \hat{\Pi}\left(q^{2}\right),
$$

where

$$
\hat{\Pi}\left(q^{2}\right)=\Pi(0)-\Pi\left(q^{2}\right)
$$

is the subtracted HVP, obtained from

$\left(q^{2} \delta_{\mu \nu}-q_{\mu} q_{\nu}\right) \Pi\left(q^{2}\right)=\int d^{4} x e^{i q x}\left\langle j_{\mu}^{\mathrm{EM}}(x) j_{\nu}^{\mathrm{EM}}(0)\right\rangle$,

with $j_{\mu}^{\mathrm{EM}}(x)$ the hadronic part of the electromagnetic current and $\alpha$ the fine-structure constant. Here the momentum $q$ is Euclidean, and throughout this paper, we will work in Euclidean space. The weight $f\left(q^{2}\right)$ in Eq. (1.1) depends on the muon mass $m_{\mu}$ and is given by

$$
\begin{aligned}
f\left(q^{2}\right) & =\frac{m_{\mu}^{2} q^{2} Z^{3}\left(q^{2}\right)\left(1-q^{2} Z\left(q^{2}\right)\right)}{1+m_{\mu}^{2} q^{2} Z^{2}\left(q^{2}\right)}, \\
Z\left(q^{2}\right) & =\frac{\sqrt{1+4 m_{\mu}^{2} / q^{2}}-1}{2 m_{\mu}^{2}} .
\end{aligned}
$$

\footnotetext{
${ }^{2}$ A small mistake was corrected in Ref. [11]; for the computation of Ref. [10] the numerical effect of this mistake is negligibly small.
}

The integral in Eq. (1.1) is finite in QCD, as can be seen from the operator product expansion of $j_{\mu}^{\mathrm{EM}}(x) j_{\nu}^{\mathrm{EM}}(0)$, which governs the behavior of $\hat{\Pi}\left(q^{2}\right)$ at large $q^{2}$. However, ChPT, which is designed to parametrize the small- $q^{2}$ behavior of $\hat{\Pi}\left(q^{2}\right)$, does not get the large- $q^{2}$ behavior right, and therefore, when we insert a ChPT representation of $\hat{\Pi}\left(q^{2}\right)$ into Eq. (1.1), the integral over $q^{2}$ does not converge beyond some order. In fact, at $k$-loop order in ChPT,

$$
\hat{\Pi}\left(q^{2}\right) \sim\left(q^{2}\right)^{k-1}, \quad k=1,2, \ldots,
$$

modulo logarithmic corrections. Because $f\left(q^{2}\right) \sim m_{\mu}^{4} / q^{6}$ for large $q^{2}$, this means that the ChPT result for $a_{\mu}^{\mathrm{HVP}}$ is finite only up to NNLO, i.e., $k=2$, while at $\mathrm{N}^{3} \mathrm{LO}$ and higher the integral in Eq. (1.1) is UV divergent, and new counterterms need to be introduced to render the result finite. Such counterterms introduce new low-energy constants (LECs), and thus ChPT cannot be used to estimate $a_{\mu}^{\mathrm{HVP}}$ quantitatively beyond two-loop order, unless it would be possible to estimate the value of the (renormalized) LECs from some other physical processes.

A key point is that these new counterterms arise because of the integral over $q^{2}$ in Eq. (1.1), and they are thus not part of the ChPT Lagrangian used to calculate $\Pi\left(q^{2}\right)$ to any given order. They will necessarily be constructed not only from the pion fields of ChPT but also from the muon and photon fields. As the photon is massless, and the muon is lighter than a pion, this raises the additional question whether it is consistent to consider only FV effects associated with pions, while muons and photons are kept in an infinite volume.

In Ref. [10] we claimed that, nevertheless, pionic FV corrections can be computed in ChPT to all orders, based on the notion that FV effects refer to the IR behavior of the theory, while counterterms fix the UV behavior. As we will see, the way this separation of scales works is subtle, and the claim is perhaps not obvious. Also in a finite volume $\hat{\Pi}\left(q^{2}\right)$ takes the form of an expansion in powers of $q^{2}$ in ChPT, and one might fear that thus also the FV part of $a_{\mu}^{\mathrm{HVP}}$, defined as in Eq. (1.1) with $\hat{\Pi}\left(q^{2}\right)$ replaced by its FV part, diverges beyond NNLO in ChPT. ${ }^{3}$ This concern, that the computation of higher-order FV corrections to $a_{\mu}^{\mathrm{HVP}}$ in ChPT might break down, was raised in Ref. [15].

In this paper, we will argue that, nevertheless, FV corrections to $a_{\mu}^{\mathrm{HVP}}$ can be computed at any order in ChPT. More precisely, our claim is that, once all counterterms needed to make $a_{\mu}^{\mathrm{HVP}}$ finite in a ChPT calculation in infinite volume have been introduced, also the finitevolume corrections to $a_{\mu}^{\mathrm{HVP}}$ will be UV finite, with no

\footnotetext{
${ }^{3}$ We will argue that, in fact, the FV part of Eq. (1.1) starts diverging at $\mathrm{N}^{4} \mathrm{LO}$.
} 
need to introduce any further counterterms. In addition, this also holds when only the hadronic part of Eq. (1.1) is considered in a finite volume, while the muons and photons [the QED part of Eq. (1.1)] are kept in infinite volume. This is actually the situation encountered in lattice QCD computations of $a_{\mu}^{\mathrm{HVP}}$, in which only $\hat{\Pi}\left(q^{2}\right)$ is calculated on the lattice and thus in a finite volume.

It is beyond the scope of this paper to give an all-order proof, or even to carry out explicit ChPT calculations beyond NNLO. Instead, we will present general arguments supporting our claim and discuss the form of the new counterterms introduced to absorb UV divergences which arise in a ChPT calculation of the $q^{2}$ integral in Eq. (1.1) in more detail. This is done in Sec. II. Then, in Sec. III, we invent a toy model that allows us to demonstrate how our claim works already at two-loop order, thus illustrating the mechanism underlying our claim in a simple example. We end with our conclusions.

There are two Appendices. In the first Appendix, we discuss the relation between counterterms in the momentum representation for $a_{\mu}^{\mathrm{HVP}}$, Eq. (1.1), which is exclusively used in the main text, and the "time-momentum" representation [20] often employed in lattice computations of $a_{\mu}^{\mathrm{HVP}}$. The other Appendix contains a number of technical details needed for Secs. II and III.

\section{COUNTERTERMS FOR $a_{\mu}^{\mathrm{HVP}}$}

In Sec. II A, we begin with a qualitative discussion of the problem, based on a diagrammatic picture, explaining the necessary introduction of counterterms not present in the low-energy pion effective theory. Then, in Sec. II B, we discuss the explicit form of these counterterms in more detail, and in Sec. II C we present illustrative examples of the role of these counterterms. We will have a first look at FV effects in Sec. II D, to argue that the interplay between UV divergences and FV effects only becomes nontrivial at $\mathrm{N}^{4} \mathrm{LO}$. While FV effects will also be qualitatively discussed in Sec. II A below, most of the finite-volume discussion will be postponed to Sec. III; the bulk of this section will concentrate on the counterterm structure for $a_{\mu}^{\mathrm{HVP}}$ in infinite volume.

\section{A. Qualitative discussion}

In Fig. 1, diagram N0 depicts the standard diagrammatic picture of the HVP contribution to the muon anomalous magnetic moment. According to Refs. [14,15], the HVP contribution in Eq. (1.1) may, in more detail, be thought of in terms of a forward pion Compton scattering subdiagram. This is schematically depicted in panel N1 of Fig. 1 where the internal Compton amplitude is obtained by cutting open the pion loop. In this diagram the fat line depicts any number of pions as well as any other heavier hadrons, such as, e.g., the $\rho, \omega$, etc., resonance contributions or a proton loop, which are denoted in the diagram by $H$. In this picture, ChPT is the result of "integrating out" all heavier states denoted by $H$, giving rise to the low-energy EFT which is ChPT. Although it is not known how to make this quantitative in the real world, it presents a clear picture which will prove helpful in what follows.

Diagram N1 ${ }^{\mathrm{EFT}}$ schematically depicts the contribution of the low-energy degrees of freedom, i.e., the pions, while diagrams N2-N5 originate from integrating out all heavier hadronic contributions, collectively denoted as $H$ in diagram N1. As the $H$ states are considered infinitely heavier than the pions in the setting of the EFT, all $H$ propagators get shrunk to a point, as seen, e.g., in diagram N2. Only pion loops remain, with one of them schematically indicated in $\mathrm{N} 2$. The sum of $\mathrm{N} 1{ }^{\mathrm{EFT}}$ and $\mathrm{N} 2$ has a different $\mathrm{UV}$ behavior than diagram N1, which must be compensated by the necessary low-energy constants which play the role of counterterms to subtract the UV divergences which are produced by the "incorrect" large-momentum behavior of the sum of $\mathrm{N} 1{ }^{\mathrm{EFT}}$ and $\mathrm{N} 2$. These counterterm contributions correspond to the diagrams denoted by N3-N5. Both the loop containing the muon line as well as pion loops become more divergent as a consequence of contracting heavyhadron propagators to a point. Diagram N3 renormalizes the UV subdivergence from the muon-photon loop in $\mathrm{N} 1{ }^{\mathrm{EFT}}$ and $\mathrm{N} 2{ }^{5}$ while $\mathrm{N} 4$ renormalizes the UV subdivergence from the pion loop in N1 ${ }^{\mathrm{EFT}}$ and N2. Diagram N5 is needed to renormalize the product of these divergences. Each of these new "vertices" (denoted by solid squares in diagrams N2-N5) corresponds to a set of new higherdimension operators suppressed by the right power of the scale $M_{H}$ characterizing the heavy states $H$. In this sense, ChPT is nothing but an expansion in inverse powers of $M_{H}$. As is clear from these diagrams, one should expect not only an LEC associated with the HVP subdiagram (N4) but also a mixed one involving pions, muons and photons (N3), as well as a counterterm without pions (N5). Integrating out $H$, therefore, yields an EFT which is not just ChPT but ChPT enlarged by the presence of muons and photons; in other words, what we need is the EFT for low-energy QCD plus QED. Diagram N4 corresponds to a counterterm in standard ChPT, but both N3 and N5 correspond to Paulilike counterterms coupling to the muon and the photon, with N3 coupling to two pions as well.

So far, our discussion has been restricted to infinite volume, and we now turn to the implications for the EFT in a finite volume.

While the analysis in this paper will be carried out in momentum space, it is useful to think about the origin of FV effects in position space. Pion-induced FV corrections

\footnotetext{
${ }^{4}$ While the diagrams show only one pion loop explicitly, there can be more than one pion loop.

${ }^{5}$ While the muon-photon(-pion) loop in $\mathrm{N} 1{ }^{\mathrm{EFT}}$ and N2 looks convergent, at sufficiently high order in ChPT there will be derivatives at the photon-pion vertex making this loop divergent.
} 


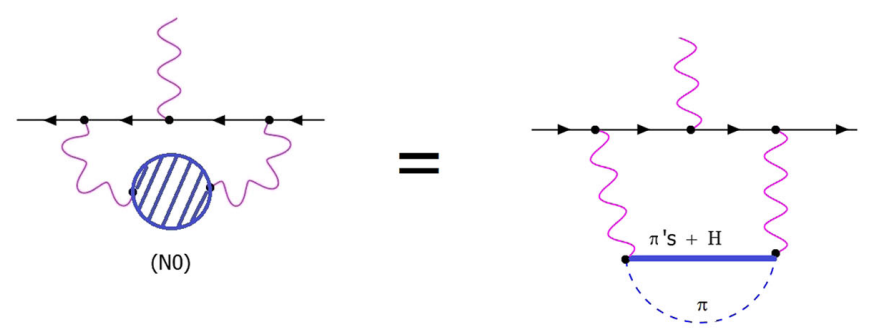

(N1)

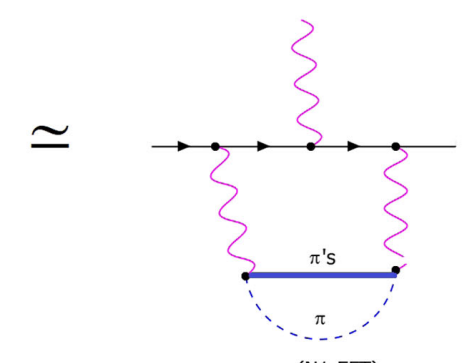

(N1-EFT)

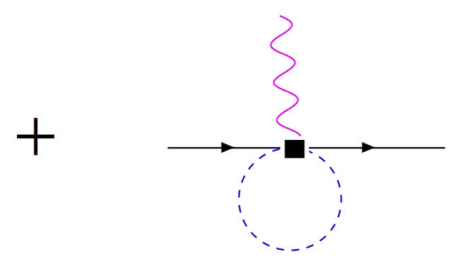

(N3)

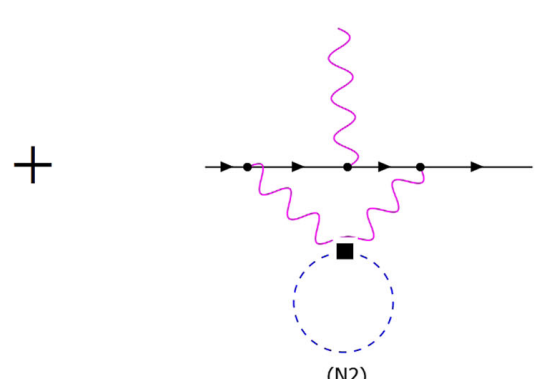

(N2)

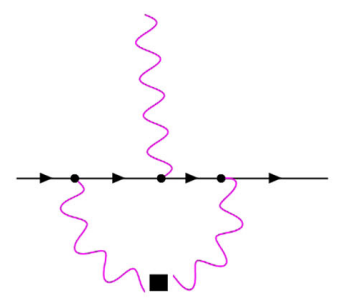

(N4)

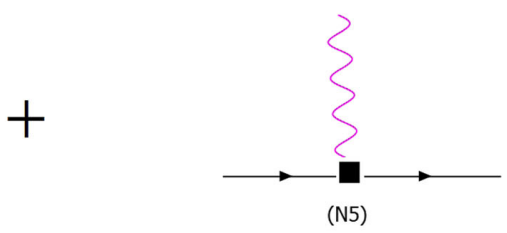

FIG. 1. $a_{\mu}^{\mathrm{HVP}}$ in terms of hadronic Feynman diagrams.

to the HVP are obtained by having (at least) one pion wrap (at least once) around the "periodic world," thus producing a factor $e^{-n m_{\pi} L}$, where $L$ is the linear size of the periodic spatial volume [12]. Here $n$ is a number relating to how many times in which directions the pion wraps around the world: if the pion wraps once in one direction, $n=1$; if it wraps once in two different directions, $n=\sqrt{2}$, etc. Since a pion wrapping at least once around the world travels a long distance, it will not contribute to the degree of divergence of the diagram. However, the remainder of the diagram may still be UV divergent, and one may thus end up with a FV correction multiplied by a UV divergence. This appears to be in conflict with the claim that FV corrections can be systematically computed in an EFT framework. As we will see explicitly in Sec. III, in momentum space, forcing a pion to wrap around the periodic world corresponds to cutting the corresponding pion loop, i.e., putting that pion on shell.
Within ChPT, there will be a counterterm corresponding to the divergent subdiagram. At orders beyond the order at which the counterterm first appears, it will contribute to loop diagrams as well, and again cutting pion lines on these loops will also lead to FV corrections of the same form, multiplied by the LEC associated with the counterterm. Since this LEC is UV divergent, this will renormalize the coefficient of the FV correction, and the complete FV correction will turn out to be finite, thus exhibiting the expected separation of UV and IR effects in the EFT. FV effects in $\hat{\Pi}\left(q^{2}\right)$ are thus predicted in terms of the LECs of ChPT. An explicit example of this appears in Ref. [10] at NNLO, where the $\mathcal{O}\left(p^{4}\right)$ counterterm $\ell_{6}$ appears inside a one-loop diagram. The pion line in this diagram can wrap around the world, and thus the diagram contributes to FV effects, yielding a FV contribution proportional to $\ell_{6}$. But, $\mathrm{FV}$ effects also appear in the two-loop diagrams that appear at this order. Taking the pion on one of the loops around the 
world still leaves a one-loop subdivergence. This one-loop subdivergence is renormalized by $\ell_{6}$, and the sum of the $\mathrm{FV}$ effects coming from the two-loop diagrams and the oneloop diagrams containing $\ell_{6}$ is UV finite. The vertex corresponding to the counterterm with LEC $\ell_{6}$ is an example of a diagram of type N4 in Fig. 1.

In the case of $a_{\mu}^{\mathrm{HVP}}$, there appear also counterterms corresponding to the black squares in diagrams N3 and N5. The reason for this is as follows. $a_{\mu}^{\mathrm{HVP}}$ is proportional to the projection on the Dirac structure associated with the muon form factor $F_{2}\left(q^{2}\right)$ at low momentum of a correlation function in QCD coupled to muons and photons. This enlarged theory is described at low energy by a theory of pions coupled to muons and photons (which, both being lighter than pions, have to be kept as explicit degrees of freedom in the EFT). In this extended EFT, new counterterms can appear which contain muon and photon fields combining into a Pauli-like operator. While in the renormalizable UV-complete theory consisting of QCD plus QED such a counterterm cannot (and does not) appear since it has at least dimension five, in the EFT there is no restriction on the dimension of possible counterterms, because an arbitrary inverse power of the heavy scale $M_{H}$ that has been integrated out can appear multiplying these counterterms. Pauli-like counterterms are thus expected to appear. In the next subsection, we will consider the explicit form of such counterterms.

\section{B. Pauli-like counterterms}

In this section, we consider the explicit form of counterterms corresponding to diagrams N3 and N5 in Fig. 1. Counterterms corresponding to diagram N4 are those already appearing in the EFT for pions only. At NNLO, examples of the LECs associated with N4-type counterterms are the $\mathcal{O}\left(p^{4}\right)$ LEC $\ell_{6}$ (for its role in the ChPT approach to $a_{\mu}^{\mathrm{HVP}}$ see Ref. [10]) and the $\mathcal{O}\left(p^{6}\right)$ LEC $c_{56}$ [21] (see Appendix A); their role is to renormalize the HVP subdiagram of diagram N1 ${ }^{\mathrm{EFT}}$ in Fig. 1. Here, instead, we will construct the simplest example of a counterterm in the EFT which includes also muons and photons.

At $\mathrm{N}^{3} \mathrm{LO}, \hat{\Pi}\left(q^{2}\right) \sim q^{4}$ modulo logarithmic corrections, and, since $f\left(q^{2}\right) \sim m_{\mu}^{4} / q^{6}$, the integral in Eq. (1.1) diverges at this order, requiring an N5-type counterterm. Of course, the counterterm that is needed must be proportional to

$$
\bar{\psi}_{\mu} \sigma_{\kappa \lambda} \psi_{\mu} F_{\kappa \lambda}
$$

where $\psi_{\mu}$ is the muon field and $F_{\kappa \lambda}$ is the electromagnetic field strength. However, we will also need N3-type counterterms, and in general, in the theory with pions, muons and photons, we need to analyze the general structure N3- and N5-type counterterms can have. The standard method for carrying out this analysis is through the use of spurions.
We start with coupling massless, two-flavor QCD to $[S U(2) \times U(1)]_{L} \times[S U(2) \times U(1)]_{R}$ vector sources $\ell_{\kappa}$ and $r_{\kappa}$, which we will eventually set equal to the photon field $A_{\kappa}$ by choosing

$$
\ell_{\kappa}=-\frac{1}{2}\left(1-\tau_{3}\right) A_{\kappa}, \quad r_{\kappa}=-\frac{1}{2}\left(1-\tau_{3}\right) A_{\kappa},
$$

where $\tau_{i}$ are the Pauli matrices. We also introduce a muon doublet, $\psi=\left(\psi_{\nu}, \psi_{\mu}\right)^{T}$, which couples to the spurions $\ell_{\kappa}$ and $r_{\kappa}$ through

$$
\bar{\psi} \gamma_{\kappa}\left(\partial_{\kappa}+i \ell_{\kappa} P_{L}+i r_{\kappa} P_{R}\right) \psi
$$

where $P_{R}$ and $P_{L}$ are right- and left-handed projectors, respectively. The nonlinear pion field $U=\exp \left[2 i \pi / f_{\pi}\right]$ couples to the spurions through the covariant derivative

$$
D_{\kappa} U=\partial_{\kappa} U+i \ell_{\kappa} U-i U r_{\kappa} .
$$

Since, for large momenta, the weight $f\left(q^{2}\right)$ is proportional to $m_{\mu}^{4}$, the N3- and N5-type counterterms have to contain the third power of the muon mass, ${ }^{6}$ and we thus need to introduce a spurion $\chi^{(\mu)}$ for the muon mass as well. Finally, since $a_{\mu}^{\mathrm{HVP}}$ contains internal photon lines, we need the charge matrix spurions $Q_{L}$ and $Q_{R}$ through which the photon couples to the left-handed and right-handed quarks, respectively. Of course, $Q_{L}=Q_{R}=Q=\operatorname{diag}\left(\frac{2}{3},-\frac{1}{3}\right)$, but the spurions $Q_{L}$ and $Q_{R}$ transform differently, under $[S U(2) \times U(1)]_{L}$ and $\left.[S U(2) \times U(1)]_{R}\right]$, respectively. QCD coupled to the muon doublet $\psi$ and the spurions $\ell_{\kappa}, r_{\kappa}$ and $\chi^{(\mu)}$, and thus our EFT, is invariant under ${ }^{7}$

$$
\begin{aligned}
U & \rightarrow L U R^{\dagger}, \\
\psi & \rightarrow\left(L P_{L}+R P_{R}\right) \psi, \\
\ell_{\kappa} & \rightarrow L \ell_{k} L^{\dagger}-i L \partial_{\kappa} L^{\dagger}, \\
r_{\kappa} & \rightarrow R r_{k} R^{\dagger}-i R \partial_{\kappa} R^{\dagger}, \\
\chi^{(\mu)} & \rightarrow L \chi^{(\mu)} R^{\dagger}, \\
Q_{L} & \rightarrow L Q_{L} L^{\dagger}, \\
Q_{R} & \rightarrow R Q_{R} R^{\dagger},
\end{aligned}
$$

where $L \in[S U(2) \times U(1)]_{L}$ and $R \in[S U(2) \times U(1)]_{R}$. For a complete construction of the EFT also a spurion $\chi^{(\pi)}$ for the quark mass transforming in the same way as $\chi^{(\mu)}$ would be needed, but we will not need it for the counterterms discussed below.

\footnotetext{
${ }^{6}$ One factor $m_{\mu}$ in $f\left(q^{2}\right)$ comes from the definition of $a_{\mu}^{\mathrm{HVP}}$ and not from diagram N0.

${ }^{7}$ We ignore the $U(1)$ axial anomaly, because the corresponding source will be set equal to zero.
} 
Ignoring the pions for the moment, the simplest counterterm leading to the Pauli structure (2.1) is

$$
\begin{aligned}
& \bar{\psi}_{L} \sigma_{\kappa \lambda} \chi^{(\mu)} r_{\kappa \lambda} \psi_{R}+\bar{\psi}_{R} \sigma_{\kappa \lambda} r_{\kappa \lambda} \chi^{(\mu) \dagger} \psi_{L}+\bar{\psi}_{R} \sigma_{\kappa \lambda} \chi^{(\mu) \dagger} \ell_{\kappa \lambda} \psi_{L} \\
& \quad+\bar{\psi}_{L} \sigma_{\kappa \lambda} \ell_{\kappa \lambda} \chi^{(\mu)} \psi_{R} .
\end{aligned}
$$

At least one power of $\chi^{(\mu)}$ is needed, consistent with the fact that one factor $m_{\mu}$ has to appear because of the helicity flip of the muon associated with its magnetic moment. Since we need the third power of the muon mass, two more spurion factors $\chi^{(\mu)}$ or $\chi^{(\mu) \dagger}$ need to be inserted. This can be done in various ways consistent with the symmetry (2.5), but they all collapse to the same factor $m_{\mu}^{3}$ once we set $\chi^{(\mu)}=m_{\mu}$.

The invariant operator with the lowest dimension involving the pion field $U$ is

$$
\operatorname{tr}\left(Q_{L} U Q_{R} U^{\dagger}\right)
$$

where $Q_{L}$ and $Q_{R}$ appear because of the internal photon lines in diagram N0. Multiplying Eq. (2.6) with two more insertions of the spurion $\chi^{(\mu)}$ with Eq. (2.7), setting $\chi^{(\mu)}=m_{\mu}, \ell_{\kappa}$ and $r_{\kappa}$ equal to the values in Eq. (2.2) and $Q_{L}=Q_{R}=Q$, we obtain the counterterm

$$
\begin{aligned}
& \frac{\alpha^{2} m_{\mu}^{3}}{\left(4 \pi f_{\pi}\right)^{4}} F_{\kappa \lambda} \bar{\psi}_{\mu} \sigma_{\kappa \lambda} \psi_{\mu} \operatorname{tr}\left(Q_{L} U Q_{R} U^{\dagger}\right) \\
& =\frac{\alpha^{2} m_{\mu}^{3}}{\left(4 \pi f_{\pi}\right)^{4}} F_{\kappa \lambda} \bar{\psi}_{\mu} \sigma_{\kappa \lambda} \psi_{\mu}\left(\frac{5}{9}-\frac{4}{f_{\pi}^{2}} \pi^{+} \pi^{-}+\mathcal{O}\left(\pi^{4}\right)\right) .
\end{aligned}
$$

We multiplied with the factor $1 /\left(4 \pi f_{\pi}\right)^{4}$ to make this a dimension-four operator, with the scale $4 \pi f_{\pi}$ standing in for the hadronic scale $M_{H}$ of the "heavy" hadrons that have been integrated out. The two powers of $\alpha$ reflect the presence of the two internal photon lines. The fact that the pion fields are traced over corresponds to the fact that they appear in a loop; if there were $n$ pairs of charged pions, their contribution to $a_{\mu}^{\mathrm{HVP}}$ would be $n$ times larger. ${ }^{8}$ Furthermore, it is clear that the square of the quark charge matrix has to appear from the quark picture of the HVP.

Setting the pion field $\pi=0$ in Eq. (2.8), this counterterm is of the form (2.1) and thus is the simplest example of an N5-type counterterm, with its coefficient renormalizing the overall divergence that can appear in the EFT calculation of Eq. (1.1). Since such a divergence appears for the first time at $\mathrm{N}^{3} \mathrm{LO}$, we expect this counterterm to be of order $1 /\left(4 \pi f_{\pi}\right)^{4}$. Indeed, dimensional analysis leads to the appearance of this factor in Eq. (2.8), and the counterterm thus takes a natural form. Of course, divergences also appear beyond $\mathrm{N}^{3} \mathrm{LO}$, and corresponding counterterms

\footnotetext{
${ }^{8}$ Indeed, other charged mesons, such as the $K^{+} K^{-}$pair, do contribute, but their contribution is suppressed because of the larger mass of these mesons.
}

proportional to powers of $1 /\left(4 \pi f_{\pi}\right)$ larger than four will be needed as well. Such counterterms are easily constructed by inserting (covariant) derivatives and/or powers of the pion mass.

The counterterm (2.8) also produces a counterterm of order $1 /\left(4 \pi f_{\pi}\right)^{6}$ with a photon, two muon and two pion external lines, and such a counterterm leads to diagrams of type N3 in Fig. 1 . The power of $1 /\left(4 \pi f_{\pi}\right)$ thus suggests that this counterterm will only be needed at $\mathrm{N}^{4} \mathrm{LO}$. Moreover, only N3-type (and not N5-type) counterterms will contribute to pion-induced FV effects. This is consistent if the first UV-divergent FV effects requiring an N3-type counterterm only appear at $\mathrm{N}^{4} \mathrm{LO}$, so that they are renormalized by the N3-type counterterm in Eq. (2.8). As we will see in Sec. III, in order to produce a FV effect, a pion line needs to be cut. This makes it plausible that no UV-divergent FV effects occur at three-loop order, because cutting one pion line reduces the degree of divergence. While a full three-loop calculation is beyond the scope of this paper, in the next subsection we give a more quantitative argument supporting this conjecture.

We close this subsection with a comment on Ref. [15], to point out that the discussion above is in agreement with the power counting in that reference. In footnote 3 of Ref. [15], it is stated that an $\mathrm{N}^{3} \mathrm{LO}$ calculation of the Compton scattering amplitude, which is obtained as a subdiagram of diagram N1 in Fig. 1 by cutting the dashed pion line, would lead to a divergent contribution to the FV effects in $\alpha_{\mu}^{\mathrm{HVP}}$. To turn the Compton scattering amplitude into the HVP, one has to close the pion loop, as in diagram N1. Because of the extra loop, this turns the diagram into an $\mathrm{N}^{4} \mathrm{LO}$ contribution to the HVP. Thus, one expects this divergence to be canceled by an $\mathrm{N}^{4} \mathrm{LO}$ counterterm, which is precisely in agreement with what we found above.

\section{An explicit example}

A very simplified model for the "three-loop" HVP is given by

$$
\Pi_{0}\left(q^{2}\right)=\frac{q^{4}}{f_{\pi}^{4}} \int d^{4} p \frac{1}{p^{2}+m_{\pi}^{2}} \frac{1}{(p-q)^{2}+m_{\pi}^{2}} .
$$

Obviously this is clearly not a true three-loop contribution. However, in accordance with Eq. (1.5), this model "threeloop" HVP behaves like $\mathcal{O}\left(q^{4} \log q^{2}\right)$ and is divergent, and these are the essential ingredients we need for our discussion. ${ }^{9}$

Regulating the integral as $d^{4} p \rightarrow d p^{2} p^{2}\left(p^{2} / \mu^{2}\right)^{\epsilon}$, thus introducing the unphysical scale $\mu$, and ignoring numerical factors from angle integrations, etc., the result is

\footnotetext{
${ }^{9}$ Of course, at three loops more complicated logarithmic corrections to the $q^{4}$ behavior can appear, but we believe this is not essential to the point we wish to make.
} 


$$
\begin{aligned}
\Pi_{0}\left(q^{2}\right) & =\frac{q^{4}}{f_{\pi}^{4}} \int_{0}^{1} d x\left(-1-\frac{1}{\epsilon}-\log \frac{x(1-x) q^{2}+m_{\pi}^{2}}{\mu^{2}}\right) \\
& =\frac{q^{4}}{f_{\pi}^{4}}\left(-\frac{1}{\epsilon}+1-\log \frac{m_{\pi}^{2}}{\mu^{2}}+\frac{\sqrt{4 m_{\pi}^{2}+q^{2}}}{q} \log \frac{\sqrt{4 m_{\pi}^{2}+q^{2}}-q}{\sqrt{4 m_{\pi}^{2}+q^{2}}+q}\right) .
\end{aligned}
$$

Adding the "ChPT" counterterm

$$
\Pi_{\mathrm{CT}}\left(q^{2}\right)=\frac{q^{4}}{f_{\pi}^{4}}\left(\frac{1}{\epsilon}+\ell(\mu)-1\right),
$$

in order to subtract the divergence in Eq. (2.10), and allowing for an additional finite renormalization $\frac{q^{4}}{f_{\pi}^{4}}(\ell(\mu)-1)$, one obtains the renormalized "three-loop" pion vacuum polarization

$$
\begin{aligned}
\hat{\Pi}_{0}\left(q^{2}\right) & \equiv \Pi_{0}\left(q^{2}\right)+\Pi_{\mathrm{CT}}\left(q^{2}\right) \\
& =\frac{q^{4}}{f_{\pi}^{4}}\left(\ell(\mu)-\int_{0}^{1} d x \log \frac{x(1-x) q^{2}+m_{\pi}^{2}}{\mu^{2}}\right) \\
& =\frac{q^{4}}{f_{\pi}^{4}}\left(\ell(\mu)-\log \frac{m_{\pi}^{2}}{\mu^{2}}+\frac{\sqrt{4 m_{\pi}^{2}+q^{2}}}{q} \log \frac{\sqrt{4 m_{\pi}^{2}+q^{2}}-q}{\sqrt{4 m_{\pi}^{2}+q^{2}}+q}\right) .
\end{aligned}
$$

The renormalized quantity $\hat{\Pi}_{0}\left(q^{2}\right)$ is a physical quantity and thus should not depend on the unphysical scale $\mu$. Therefore, in this simple example the running of the renormalized LEC $\ell(\mu)$ satisfies

$$
\mu \frac{d}{d \mu} \ell(\mu)=-2 .
$$

Since the model of Eq. (2.9) is not UV complete, we cannot determine the dependence of $\ell(\mu)$ on the UV physics that has been integrated out, and the running of $\ell(\mu)$ in (2.13) is all we can know. In Sec. III we will study a simple model which is UV complete.

As a model for the contribution to the muon anomaly from $\hat{\Pi}_{0}\left(q^{2}\right)$ we will choose the model "anomaly" $a$ to be given by the integral $\left(m \equiv m_{\mu}\right)$

$$
a=m^{4} \int \frac{d^{n} q}{q^{2}+m^{2}} \frac{1}{q^{6}} \hat{\Pi}_{0}\left(q^{2}\right)\left(\frac{M^{2}}{q^{2}+M^{2}}\right),
$$

where $1 / q^{6}$ represents the combination of photon propagators and other kinematical factors and $1 /\left(q^{2}+m^{2}\right)$ regulates the IR divergence (i.e., it gives the dependence on the muon mass in our simplified representation). The factor $M^{2} /\left(q^{2}+M^{2}\right)$ has been inserted to make the $q$ integral in Eq. (2.14) finite and "stands in" for the nonpionic hadron physics of QCD. One might think of it as the insertion of a fake $\rho$ propagator, but this is not essential: the only job of this factor is to regulate the UV divergence of the $q$ integral. As we will see, it will allow us to determine the form of the counterterm needed to make $a$ finite without this factor.

As it stands, the integral (2.14) is finite and well behaved for $d=4$. The "ChPT" version of $a$ is obtained by setting $M^{2} /\left(q^{2}+M^{2}\right) \rightarrow 1$, i.e., sending $M^{2} \rightarrow \infty$, but of course, that reintroduces the UV divergence of the $q$ integral.

In order to proceed, we split

$$
\frac{M^{2}}{q^{2}+M^{2}}=1-\frac{q^{2}}{q^{2}+M^{2}},
$$

and using the expression for $\hat{\Pi}_{0}\left(q^{2}\right)$ in Eq. (2.12) one may split the contributions to $a$ as

$$
\begin{aligned}
a= & a_{\mathrm{EFT}}+a_{\mathrm{CT}} \\
= & \frac{m^{4}}{f_{\pi}^{4}} \int_{0}^{\infty} \frac{d q^{2}}{q^{2}+m^{2}}\left(\frac{q^{2}}{\mu^{2}}\right)^{\epsilon}\left(\ell(\mu)-\int_{0}^{1} d x \log \frac{q^{2} x(1-x)+m_{\pi}^{2}}{\mu^{2}}\right) \\
& -\frac{m^{4}}{f_{\pi}^{4}} \int_{0}^{\infty} \frac{d q^{2}}{q^{2}+\underbrace{m^{2}}_{\text {neglect }}}\left(\frac{q^{2}}{\mu^{2}}\right)^{\epsilon} \frac{q^{2}}{q^{2}+M^{2}}\left(\ell(\mu)-\int_{0}^{1} d x \log \frac{q^{2} x(1-x)+m_{\pi}^{2}}{\mu^{2}}\right),
\end{aligned}
$$


where, as indicated, the muon mass $m$ may be neglected in the second integral, $a_{\mathrm{CT}}$, on account of the extra $q^{2}$ in the numerator. As we will see, this also allows the limit $m_{\pi}^{2} \rightarrow 0$ to be taken, rendering $a_{\mathrm{CT}}$ independent of the IR scales $m$ and $m_{\pi}$, as expected for a counterterm.

The integrals yield cumbersome expressions for $m \neq m_{\pi}$. This is why we will simplify our example by taking $m=m_{\pi}$ as a common low-energy scale, $m=m_{\pi} \ll M$, in the rest of this section. This simplification only serves to simplify the math and is not essential, of course.

Setting $m_{\pi}=m$, the result for $a_{\mathrm{EFT}}$ can be obtained by evaluating the integrals in Eq. (2.16a):

$$
\begin{aligned}
a_{\mathrm{EFT}} & =\frac{m^{4}}{f_{\pi}^{4}}\left[\ell(\mu)\left(-\frac{1}{\epsilon}-\log \frac{m^{2}}{\mu^{2}}\right)\right. \\
& \left.-\frac{1}{\epsilon^{2}}-\frac{2}{\epsilon}-2 \log \frac{m^{2}}{\mu^{2}}+\frac{1}{2} \log ^{2} \frac{m^{2}}{\mu^{2}}+\frac{2}{27} \pi^{2}+\mathcal{C}\right],
\end{aligned}
$$

where the first (second) line corresponds to the first (second) term in the integrand of Eq. (2.16) and $\mathcal{C}$ is a constant given by

$\mathcal{C}=-\frac{1}{9} \psi^{\prime}\left(\frac{1}{6}\right)-\frac{1}{18} \psi^{\prime}\left(\frac{1}{3}\right)+\frac{1}{9} \psi^{\prime}\left(\frac{2}{3}\right)+\frac{1}{18} \psi^{\prime}\left(\frac{5}{6}\right)$,

where $\psi^{\prime}(x)=\frac{d \psi(x)}{d x}$ with $\psi(x)$ is the digamma function $\psi(x)=\frac{\Gamma^{\prime}(x)}{\Gamma(x)}$.

We next turn to $a_{\mathrm{CT}}$ in Eq. (2.16b). The result of the integral is

$$
\begin{aligned}
a_{\mathrm{CT}}= & -\frac{m^{4}}{f_{\pi}^{4}}\left[\ell(\mu)\left(-\frac{1}{\epsilon}-\log \frac{M^{2}}{\mu^{2}}\right)\right. \\
& -\frac{1}{\epsilon^{2}}-\frac{2}{\epsilon}-2 \log \frac{M^{2}}{\mu^{2}}+\frac{1}{2} \log ^{2} \frac{M^{2}}{\mu^{2}}+\frac{\pi^{2}}{6} \\
& \left.+\mathcal{O}\left(\frac{m_{\pi}^{2}}{M^{2}} \log ^{2} \frac{m_{\pi}^{2}}{M^{2}}\right)\right],
\end{aligned}
$$

where again the first (second) line corresponds to the first (second) term in the integrand of Eq. (2.16b). We emphasize that for the second line, i.e., the term with the $\log \left(q^{2} x(1-x)+m_{\pi}^{2}\right)$, the limit $m_{\pi}^{2} \rightarrow 0$ may be taken, resulting in a term independent of $m_{\pi}^{2} \cdot{ }^{10}$ In the third line of Eq. (2.19) we have kept $m_{\pi}^{2}$ explicit for illustration, but this whole term is $\mathcal{O}\left(\frac{m_{\pi}^{2}}{M^{2}}\right)$ and therefore is to be neglected at the order we consider. In Appendix B we show how to calculate it from Eq. (2.16b).

\footnotetext{
${ }^{10}$ Dependence on $m_{\pi}$ would be provided by higher-order counterterms.
}

Adding $a_{\mathrm{EFT}}$ and $a_{\mathrm{CT}}$ in Eqs. (2.17) and (2.19) we finally obtain the result

$$
\begin{aligned}
a= & \frac{m^{4}}{f_{\pi}^{4}}\left(\ell(\mu) \log \frac{M^{2}}{m^{2}}+2 \log \frac{M^{2}}{m^{2}}\right. \\
& \left.+\frac{1}{2} \log \frac{M^{2}}{m^{2}} \log \frac{\mu^{4}}{m^{2} M^{2}}-\frac{5}{54} \pi^{2}+\mathcal{C}\right),
\end{aligned}
$$

where $\mathcal{C}$ is given in Eq. (2.18). Note how the condition (2.13) for the running of $\ell(\mu)$ makes our result independent of $\mu$.

The result for $a_{\mathrm{CT}}$ in Eq. (2.19) admits an expansion in powers of $m_{\pi}^{2} / M^{2}$. To lowest order in this expansion, there are no $\operatorname{logarithms~such}$ as $\log m_{\pi}^{2} / M^{2}$, which could only come from a pion loop. Therefore, the conclusion is that the leading order is given by an N5-type Pauli-like operator, without any pion loops. Thus at leading order (which, recall, in the real world corresponds to three-loop order), only an N5-type counterterm is needed. It is only at the next order in the chiral expansion, i.e., $\mathcal{O}\left(m_{\pi}^{2} / M^{2} \times m_{\mu}^{4} / f_{\pi}^{4}\right)$, that these logarithms appear: this is when tadpole diagrams of type N3 will start to contribute.

\section{The example in finite volume}

Let us now extend the discussion of our example to have a first look at FV effects coming from the pions. We thus want to consider the integral

$$
\begin{aligned}
D\left(q^{2}\right)= & \int_{-\infty}^{\infty} \frac{d p_{4}}{2 \pi} \int \frac{d^{3} p}{(2 \pi)^{3}} \frac{1}{p_{4}^{2}+p^{2}+m_{\pi}^{2}} \\
& \times \frac{1}{\left(p_{4}-q_{4}\right)^{2}+p^{2}+m_{\pi}^{2}}
\end{aligned}
$$

in a finite spatial volume $V=L^{3}$ of linear dimension $L$, with periodic boundary conditions. Here we took $q=$ $\left(0,0,0, q_{4}\right)$ to point in the 4-direction, without loss of generality, as this will be convenient in our explicit calculations. In finite volume, the integral over $\vec{p}$ is replaced by a sum, and in finite volume $D$ becomes

$$
\begin{aligned}
D_{\mathrm{FV}}\left(q_{4}^{2}\right)= & \int_{-\infty}^{\infty} \frac{d p_{4}}{2 \pi} \frac{1}{L^{3}} \sum_{\vec{p}=2 \pi \vec{n} / L} \frac{1}{p_{4}^{2}+p^{2}+m_{\pi}^{2}} \\
& \times \frac{1}{\left(p_{4}-q_{4}\right)^{2}+p^{2}+m_{\pi}^{2}},
\end{aligned}
$$

where $\vec{n}$ has integer components. In order to isolate the FV effects, we will use Poisson resummation:

$$
\begin{aligned}
\frac{1}{L^{3}} \sum_{\vec{p}} f\left(\vec{p}^{2}\right) & =\sum_{\vec{n}} \int \frac{d^{3} \vec{p}}{(2 \pi)^{3}} e^{i \vec{n} \cdot \vec{p} L} f\left(\vec{p}^{2}\right) \\
& =\sum_{\vec{n}}\left(\frac{1}{4 i \pi^{2} n L}\right) \int_{-\infty}^{\infty} d p p f\left(p^{2}\right) e^{i n p L},
\end{aligned}
$$


in which $n=|\vec{n}|$. In position space, the vector $\vec{n}$ represents the number of times the pion wraps around the periodic spatial volume in each direction. Thus, the term with $n=0$ corresponds to the infinite-volume part, and the $n>0$ terms separate out the FV contributions. Using Eq. (2.23), we find that

$$
\begin{aligned}
D_{\mathrm{FV}}\left(q_{4}^{2}\right) & =\sum_{\vec{n}} D^{(n)}\left(q_{4}^{2}\right), \\
D^{(n)}\left(q_{4}^{2}\right) & =\int_{-\infty}^{\infty} \frac{d p_{4}}{2 \pi} \int_{-\infty}^{\infty} \frac{d p}{2 \pi^{2}} p \frac{1}{p_{4}^{2}+p^{2}+m_{\pi}^{2}} \frac{1}{\left(p_{4}-q_{4}\right)^{2}+p^{2}+m_{\pi}^{2}} \frac{e^{i n p L}}{2 i n L} \\
& =\frac{1}{8 \pi^{2} q_{4} n L} \int_{0}^{\infty} \frac{d p_{4}}{p_{4}}\left(e^{-n L \sqrt{\left(p_{4}-q_{4} / 2\right)^{2}+m_{\pi}^{2}}}-e^{-n L \sqrt{\left(p_{4}+q_{4} / 2\right)^{2}+m_{\pi}^{2}}}\right),
\end{aligned}
$$

where we used contour integration to evaluate the integral over $p$ and we shifted $p_{4} \rightarrow p_{4}+q_{4} / 2$. For large $m_{\pi} L$, the integral over $p_{4}$ is (exponentially) dominated by the region $\left(p_{4} \pm q_{4} / 2\right)^{2} \ll m_{\pi}^{2}$, allowing us to expand the square roots for large $m_{\pi}^{2}$. For large $m_{\pi} L$ and $n>0$ we thus approximate

$$
\begin{aligned}
D^{(n)}\left(q_{4}^{2}\right) & \approx \frac{1}{8 \pi^{2} q_{4} n L} e^{-m_{\pi} n L} \int_{0}^{\infty} \frac{d p_{4}}{p_{4}}\left(e^{-\left(n L m_{\pi} / 2\right)\left[\left(p_{4}-q_{4} / 2\right)^{2} / m_{\pi}^{2}\right]}-\left(q_{4} \rightarrow-q_{4}\right)\right) \\
& =\frac{1}{8 \pi^{2} q_{4} n L} e^{-m_{\pi} n L} \pi e^{-q_{4}^{2} n L / 8 m_{\pi}} \operatorname{Erfi}\left(\sqrt{\frac{q_{4}^{2} n L}{8 m_{\pi}}}\right)
\end{aligned}
$$

where $\operatorname{Erfi}(x)$ is the imaginary error function $\operatorname{erf}(i x) / i$, which is real for real $x .^{11}$

Using the asymptotic expansion

$$
\operatorname{Erfi}(x) \approx e^{x^{2}}\left(\frac{1}{\sqrt{\pi} x}+\frac{1}{2 \sqrt{\pi} x^{3}}+\frac{3}{4 \sqrt{\pi} x^{5}}+\cdots\right) \quad(x \rightarrow \infty),
$$

we obtain, for $n>0$,

$$
\begin{aligned}
D^{(n)}\left(q_{4}^{2}\right) \approx & \frac{\sqrt{2 \pi}}{4 \pi^{2}} \frac{e^{-m_{\pi} n L}}{\left(q_{4} n L\right)^{2}} \\
& \times \sqrt{m_{\pi} n L}\left(1+\mathcal{O}\left(\frac{1}{m_{\pi} n L} ; \frac{m_{\pi} n L}{\left(q_{4} n L\right)^{2}}\right)\right) .
\end{aligned}
$$

We recall that $\Pi_{0}\left(q^{2}\right)=\frac{q^{4}}{f_{\pi}^{4}} D\left(q^{2}\right)$; cf. Eq. (2.9). Consequently, we see here an example of what we anticipated earlier in this section about the $\mathrm{N}^{3} \mathrm{LO}$ contribution to $a_{\mu}^{\mathrm{HVP}}$. The insertion of $\hat{\Pi}_{0}\left(q^{2}\right)$ into the $a$ integral, as in Eq. (2.14), leads to a divergence in infinite volume which is renormalized by the corresponding counterterm; cf. Eqs. (2.16a) and (2.16b). On the other hand, the finitevolume contribution, Eq. (2.27), when inserted into the integral (2.14) for $a$ is UV finite even for $M \rightarrow \infty .^{12}$

\footnotetext{
${ }^{11}$ In order to carry out the integral in Eq. (2.25), we regulated the pole at $p_{4}=0$ by introducing a factor $p_{4}^{e}$ in each term, then combined both terms and only at the end took $\epsilon \rightarrow 0$.

${ }^{12}$ Equation (2.27) is only valid for large $q_{4} L$. This is sufficient for our argument, as it is the large- $q$ region of the integral over $q$ in Eq. (2.14) that leads to a UV divergence.
}

Intuitively, this can be understood as follows. To obtain the leading FV correction, we take the one-pion loop in any diagram to wrap "around the world." Such a pion can be seen as an on-shell pion (as we will show explicitly in Sec. III). Effectively, one thus "removes" a loop and a pion propagator, decreasing the degree of divergence by (at least) two.

However, as we have discussed above, we expect that FV effects in the real world will lead to a divergent $a_{\mu}^{\mathrm{HVP}}$ integral at $\mathrm{N}^{4} \mathrm{LO}$. Therefore, in the next section, we will study an even simpler example which, while keeping integrals at an elementary level, does lead to divergent FV effects and allows us to illustrate the interplay of these FV effects with the corresponding UV counterterms which appear at infinite volume.

\section{A TOY MODEL}

Our goal is to investigate the interplay between UV divergences, renormalization, and FV effects in more detail. As we have argued, this interplay shows up only in full force at three and four loops in the case of $a_{\mu}^{\mathrm{HVP}}$. Therefore, in this section, we will study this interplay in a very simple model, in which we do not have to go beyond two loops in order to see this interplay at work and in which details have been kept simple enough to make explicit calculations feasible.

We will define the model in Sec. III A, where we explain how keeping things simple led us to consider this model. Then, in Sec. III B, we will essentially repeat the analysis of Sec. II for the model, in infinite volume. In Sec. III C we will demonstrate how, once the infinite-volume counterterms 
have been identified, FV corrections due to the "pions" in our model are always UV finite and thus well defined.

\section{A. Definition of the model}

We wish to construct a simple toy model with pions, muons, photons, and some "heavy strong-interaction" physics in which a quantity $a$ analogous to $a_{\mu}$ can be defined. While we really just need an integral like the one in Eq. (1.1), it is instructive to cast the model in terms of a Lagrangian and Feynman rules obtained from the Lagrangian. This will allow for a diagrammatic analysis analogous to that based on Fig. 1.

The Lagrangian for our model is ${ }^{13}$

$$
\begin{aligned}
\mathcal{L}= & \frac{1}{2}\left(\partial_{\kappa} \pi\right)^{2}+\frac{1}{2} m_{\pi}^{2} \pi^{2}+\frac{1}{2}\left(\partial_{\kappa} \sigma\right)^{2}+\frac{1}{2} m_{\sigma}^{2} \sigma^{2} \\
& +\frac{1}{2}\left(\partial_{\kappa} \psi_{\mu}\right)^{2}+\frac{1}{2} m_{\mu}^{2} \psi_{\mu}^{2}+\frac{1}{2}\left(\partial_{\kappa} A\right)^{2} \\
& +\frac{1}{2} e_{1} A \pi^{2}+\frac{1}{2} e_{2} A \psi_{\mu}^{2}+e_{3} A \sigma \pi+\frac{1}{2} g \psi_{\mu}^{2} \sigma \pi .
\end{aligned}
$$

All fields are scalars, but we can intuitively think of the massless scalar $A$ as a "photon" and the scalar $\psi_{\mu}$ as a "muon," while of course $\pi$ is our "pion."14 The stronginteraction physics is represented by the massive scalar $\sigma$, and we will thus always think of $m_{\sigma}$ as much larger than $m_{\pi}$ and $m_{\mu}$.

In this model, we define a "muon anomaly" by

$$
a=e_{1} e_{2} e_{3} g \int_{0}^{\infty} d q^{2} \frac{m_{\mu}^{2}}{m_{\mu}^{2}+q^{2}} \Pi\left(q^{2}\right)
$$

where, as in Eq. (1.1), $q^{2}>0$ is Euclidean. Here we omitted purely numerical factors from the integration over the angles of $q$, and a possible symmetry factor. In Secs. III B and III C we will omit the factor $e_{1} e_{2} e_{3} g$ as well. The function $\Pi\left(q^{2}\right)$ is given by

$$
\Pi\left(q^{2}\right)=\int \frac{d^{4} p}{(2 \pi)^{4}} \frac{1}{\left(p^{2}+m_{\sigma}^{2}\right)} \frac{1}{\left[(p-q)^{2}+m_{\pi}^{2}\right]^{2}},
$$

which can be seen by traversing around the hadronic loop in the diagram depicted in Fig. 2. We emphasize that this model for $\Pi\left(q^{2}\right)$ has been chosen to be UV finite without any further subtraction, unlike Eq. (1.2). In particular, this means that also $\Pi(0)$ is finite in the model. We could have chosen a model with $\Pi\left(q^{2}\right)$ defined by Eq. (3.3) without the square on the pion propagator, in which case $\Pi\left(q^{2}\right)$ would have been logarithmically divergent. In that case, we would have considered the UV-finite difference $\Pi\left(q^{2}\right)-\Pi(0)$, as

\footnotetext{
${ }^{13}$ All similarity with the linear sigma model is purely coincidental.

${ }^{14}$ All couplings in Eq. (3.1) except $g$ have mass dimension one.
}

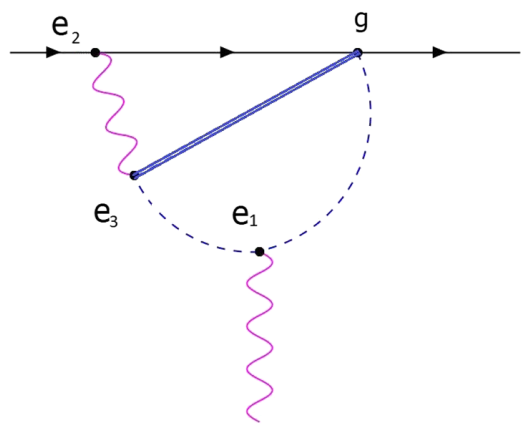

FIG. 2. Feynman diagram for the quantity $a$ defined in Eq. (3.2).

in Eq. (1.1). However, this would make the mathematical treatment of the model more cumbersome, and it is not essential, as we will see next. It is thus important to keep in mind, for the rest of this section, that $\Pi\left(q^{2}\right)$ itself is finite and does not need to be subtracted, unlike in the real world.

The diagram for the "anomaly" $a$ is shown in Fig. 2. In detail, it looks rather different from diagram N0 in Fig. 1. ${ }^{15}$ But, it shares the following essential properties with $a_{\mu}^{\mathrm{HVP}}$. First, $a$ is a UV-finite quantity, just like $a_{\mu}^{\mathrm{HVP}}$, and it is an integral over a weight function times a hadronic loop, $\Pi\left(q^{2}\right)$, which itself is finite, just like $\hat{\Pi}\left(q^{2}\right)$ in Eq. (1.1). However, as can be seen from Fig. 2, if we "integrate out" the $\sigma$, i.e., we contract its propagator to a point by replacing $1 /\left(p^{2}+m_{\sigma}^{2}\right) \rightarrow 1 / m_{\sigma}^{2}$ in Eq. (3.3), both the pion loop and the muon-photon loop become logarithmically divergent. This can be seen in diagram T2 of Fig. 3. This implies that in an EFT containing only the pions, photons and muons, while the $\sigma$ has been integrated out, counterterms will need to be introduced to renormalize these divergences. Counterterms will be needed for the pion-loop subdivergence (diagram T4), for the muon-photon-loop subdivergence (diagram T3), and the overall two-loop divergence (diagram T5). The precise form of these counterterms will be derived in Sec. III B below.

The example of the quantity $a$ in the toy model thus mimics the situation that arises at $\mathrm{N}^{3} \mathrm{LO}$ in the case of $a_{\mu}^{\mathrm{HVP}}$. At $\mathrm{N}^{3} \mathrm{LO}$ in ChPT not only are counterterms needed to renormalize UV divergences in the HVP, but also the integral over $q^{2}$ [the momentum through the muon and photon lines in Eq. (1.1)] which becomes divergent and leads to the counterterms discussed in Sec. II. Because of the construction of our model and the definition of the quantity $a$, the same phenomena happen here already at the lowest possible number of loops. Since we want to study in our model both "strong-interaction" counterterms of type N4 and "electromagnetic" counterterms of type N3 and N5,

\footnotetext{
${ }^{15}$ In fact, it bears some similarity to the hadronic light-by-light contribution to $a_{\mu}$, with the external photon line attached to the pion.
} 

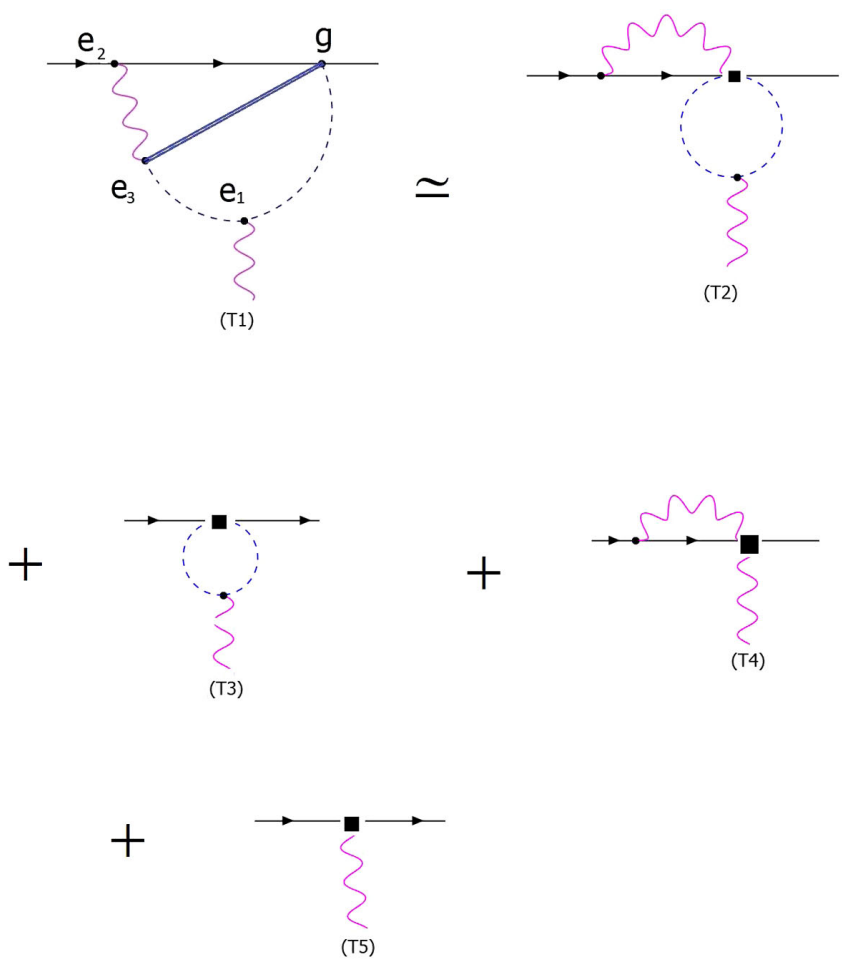

FIG. 3. $a_{\mu}^{\mathrm{HVP}}$ for the toy model.

we need a subdivergence from a pion loop and a subdivergence from a loop containing a muon, and both are present in diagram T2 of Fig. 3, which represents the EFT version of the diagram in Fig. 2 obtained by contracting the $\sigma$ propagator to a point. The detailed form of $\Pi\left(q^{2}\right)$ makes the example somewhat contrived and leads to the detailed form of the diagram in Fig. 2 and thus the diagrams in Fig. 3 to be different from the diagrams in Fig. 1. We emphasize that this is not important; what is important is the fact that the model generates both types of counterterms already at two loops. In the next subsection, we will carry out the integrals, transition to the EFT, and fully elucidate the counterterm structure in the toy model.

We end this subsection with a comment. In the model (3.1), there are other contributions to the quantity $a$, i.e., other diagrams with an external photon and two external muon lines. In particular, a contribution exists with one $\mu^{2} \sigma \pi$ and one $A \sigma \pi$ vertex, proportional to $e_{3} g$, and this contribution is UV divergent. But this will not affect our discussion of the diagram in Fig. 2. While the EFT version of the simpler oneloop contribution can be analyzed with the same method as we will use below to analyze the integral in Eq. (3.2), it does not exhibit the parallel behavior with $a_{\mu}^{\mathrm{HVP}}$ we are after. We will thus ignore this contribution and define $a$ for the rest of this section by the integral in Eq. (3.2).

\section{B. The toy model in infinite volume}

We begin with the calculation of $a$ in the complete theory, Eq. (3.1). Using Feynman parametrization of the integral in (3.3), we find (omitting, from now on, the couplings $e_{1,2,3}$ and $g$ )

$$
\begin{aligned}
\Pi\left(q^{2}\right)= & \frac{1}{16 \pi^{2}} \int_{0}^{1} d x \frac{x}{q^{2} x(1-x)+m_{\sigma}^{2}(1-x)+m_{\pi}^{2} x} \\
= & \frac{1}{16 \pi^{2}}\left(\frac{1}{m_{\sigma}^{2}+q^{2}} \log \frac{m_{\sigma}^{2}}{m_{\pi}^{2}}+\frac{m_{\sigma}^{2}-q^{2}}{q^{2}\left(m_{\sigma}^{2}+q^{2}\right)} \log \frac{m_{\sigma}^{2}}{m_{\sigma}^{2}+q^{2}}\right) \\
& +\mathcal{O}\left(\frac{m_{\pi}^{2}}{m_{\sigma}^{4}}\right) .
\end{aligned}
$$

We emphasize again that $\Pi(0)$ is finite, as can be seen by setting $q^{2}=0$ in Eq. (3.4). No subtraction is needed. In fact, we note that, to leading order in an expansion in $1 / \mathrm{m}_{\sigma}^{2}$, $\Pi\left(q^{2}\right)=\Pi(0)$. As indicated in Eq. (3.4), we will work to leading order in $m_{\pi}^{2} / m_{\sigma}^{2}$, as this will be sufficient for our purposes. Inserting this into the integral defining $a$ in Eq. (3.2), we find for $a$ the explicit result ${ }^{16}$

$$
a=\frac{m_{\mu}^{2}}{16 \pi^{2} m_{\sigma}^{2}}\left[\left(1-\log \frac{m_{\sigma}^{2}}{m_{\pi}^{2}}\right) \log \frac{m_{\mu}^{2}}{m_{\sigma}^{2}}-1+\frac{\pi^{2}}{3}+\mathcal{O}\left(\frac{m_{\mu}^{2}, m_{\pi}^{2}}{m_{\sigma}^{2}}\right)\right] .
$$

This is the result that an EFT analysis of our model is expected to reproduce, and this analysis is what we will turn to next.

The EFT for our model is obtained by integrating out the $\sigma$ field, which amounts to replacing every $\sigma$ propagator in the theory by $1 / m_{\sigma}^{2}$, thus making the exchange of a $\sigma$ meson into a point vertex. This corresponds to splitting the $\sigma$ propagator in Eq. (3.3) as

$$
\frac{1}{p^{2}+m_{\sigma}^{2}}=\frac{1}{m_{\sigma}^{2}}-\frac{p^{2}}{m_{\sigma}^{2}\left(p^{2}+m_{\sigma}^{2}\right)} \text {. }
$$

The EFT contribution corresponding to the first term on the right-hand side is depicted in diagram T2 of Fig. 3. To begin with, this replacement makes $\Pi\left(q^{2}\right)$ divergent, and a counterterm renormalizing this divergence will thus have to be introduced in the EFT. This counterterm should of course reproduce the contribution from the second term on the right-hand side of Eq. (3.6), in an expansion in inverse powers of $m_{\sigma}^{2}$. The replacement also gives rise to the divergent muon-photon loop in diagram T2; we will return to this divergence below.

As a side comment, one could study also this model at higher orders in $m_{\pi}^{2} / m_{\sigma}^{2}$ and $m_{\mu}^{2} / m_{\sigma}^{2}$ by further expanding the second term on the right-hand side of Eq. (3.6), using $-p^{2} /\left(m_{\sigma}^{2}\left(p^{2}+m_{\sigma}^{2}\right)\right)=-p^{2} / m_{\sigma}^{4}+\mathcal{O}\left(p^{4} / m_{\sigma}^{6}\right)$. But since our goal is to keep things mathematically as simple as

\footnotetext{
${ }^{16}$ One way to do the calculation is to first do perform the integral over $q^{2}$ using the Feynman-integral representation for $\Pi\left(q^{2}\right)$ of Eq. (3.4) and then carry out the integral over $x$.
} 
possible, we will only consider the expansion in inverse powers of $m_{\sigma}^{2}$ to leading order, in the rest of this paper.

Replacing $d^{4} p \rightarrow \mu^{\epsilon} d^{d} p$ with $d=4-\epsilon$ in Eq. (3.3) allows us to calculate the EFT part of $\Pi\left(q^{2}\right)$, and we find

$\Pi_{\mathrm{EFT}}\left(q^{2}\right)=\frac{1}{16 \pi^{2} m_{\sigma}^{2}}\left(\frac{2}{\epsilon}-\gamma_{E}-\log \frac{m_{\pi}^{2}}{4 \pi \mu^{2}}\right)=\Pi_{\mathrm{EFT}}(0)$.

We note that this result reproduces the $-\log m_{\pi}^{2} /\left(16 \pi^{2} m_{\sigma}^{2}\right)$ of Eq. (3.4). The toy model is the UV completion of our EFT, and thus the second term in Eq. (3.6) should lead us to the form of the counterterm. At $q=0$ we thus find the counterterm contribution by replacing the $\sigma$ propagator in Eq. (3.3) by this second term:

$\Pi_{\mathrm{CT}}(0)=-\frac{1}{16 \pi^{2} m_{\sigma}^{2}}\left(\frac{2}{\epsilon}-\gamma_{E}-\log \frac{m_{\sigma}^{2}}{4 \pi \mu^{2}}+1+\mathcal{O}\left(\frac{m_{\pi}^{2}}{m_{\sigma}^{2}}\right)\right)$.

The sum $\Pi_{\mathrm{EFT}}(0)+\Pi_{\mathrm{CT}}(0)$ reproduces $\Pi(0)$ :

$$
\Pi(0)=\frac{1}{16 \pi^{2} m_{\sigma}^{2}}\left(\log \frac{m_{\sigma}^{2}}{m_{\pi}^{2}}-1+\mathcal{O}\left(\frac{m_{\pi}^{2}}{m_{\sigma}^{2}}\right)\right) .
$$

Of course, if we do not know the underlying UV completion, we cannot calculate the contribution (3.8), as the precise form of the second term in Eq. (3.6) is not known if we only have the EFT. But, the divergent and logarithmic terms in $\Pi(0)_{\mathrm{CT}}$ can be inferred from those of $\Pi(0)_{\mathrm{EFT}}$ and dimensional analysis, as the sum of the two has to be finite and independent of $\mu$. This is precisely how a counterterm is introduced in the EFT, in order to absorb the divergence and the scale dependence that shows up in the calculation of Eq. (3.7). This does not determine the remaining finite part [here the term $\left.\left(-1+\gamma_{E}-\log 4 \pi\right) /\left(16 \pi^{2} m_{\sigma}^{2}\right)\right]$ of the counterterm. If only the EFT is known, the finite part gets replaced by an unknown finite constant of the right dimension: the value of the renormalized LEC in a particular regularization scheme (here, dimensional regularization with minimal subtraction). The counterterm discussed here takes the form

$$
\mathrm{T} 4: \frac{C_{\psi_{\mu}^{2} A^{2}}(\mu)}{4} \psi_{\mu}^{2} A^{2}
$$

and corresponds to the black square in diagram T4 in Fig. 3.

We now return to the EFT calculation of $a$ in Eq. (3.2). Diagram T2 has more divergences for which counterterms need to be introduced, and we aim to identify those from splitting $a$ into a low-energy part $a_{1}$ and a counterterm part $a_{2}$, following the same reasoning as above for $\Pi\left(q^{2}\right)$ :

$$
\begin{aligned}
a & =\int_{0}^{\infty} d q^{2}\left(\frac{q^{2}}{\mu^{2}}\right)^{\epsilon} \frac{m_{\mu}^{2}}{q^{2}+m_{\mu}^{2}}\left\{\Pi_{\mathrm{EFT}}(0)+\Pi_{\mathrm{CT}}(0)+\left[\Pi\left(q^{2}\right)-\Pi(0)\right]\right\} \\
& =a_{1}+a_{2} \\
& =\underbrace{m_{\mu}^{2} \int_{0}^{\infty} \frac{d q^{2}}{q^{2}+m_{\mu}^{2}}\left(\frac{q^{2}}{\mu^{2}}\right)^{\epsilon}\left\{\Pi_{\mathrm{EFT}}(0)+\Pi_{\mathrm{CT}}(0)\right\}}_{a_{1}}+\underbrace{m_{\mu}^{2} \int_{0}^{\infty} \frac{d q^{2}}{q^{2}+\underbrace{m_{\mu}^{2}}_{\text {neglect }}}\left(\frac{q^{2}}{\mu^{2}}\right)^{\epsilon}\left\{\Pi\left(q^{2}\right)-\Pi(0)\right\}}_{a_{2}} .
\end{aligned}
$$

Since we want to split $a$ into two divergent parts, $a_{1}$ and $a_{2}$, we introduced a regulating factor $\left(q^{2} / \mu^{2}\right)^{\epsilon}$, necessary to define each of the terms $a_{1}$ and $a_{2}$ separately; of course, $a$ itself is finite in the limit $\epsilon \rightarrow 0$. We emphasize that this regulator is independent of the regulator used in the calculation of $\Pi_{\mathrm{EFT}}(0)$ and $\Pi_{\mathrm{CT}}(0)$ in Eqs. (3.7) and (3.8) (the sum of which is finite); the use of dimensional regularization again here is just for calculational simplicity. The $m_{\mu}^{2}$ in the denominator of the integral defining $a_{2}$ may be neglected because $\Pi\left(q^{2}\right)-\Pi(0) \sim q^{2}$ as $q^{2} \rightarrow 0$. Indeed, while $a_{1}$ will depend on $m_{\mu}^{2}$ logarithmically, $a_{2}$ is analytic in $m_{\mu}^{2}$ to $\mathcal{O}\left(m_{\mu}^{2}\right)$, which is the order to which we have constructed the EFT. ${ }^{17}$ The contribution $a_{1}$ is what one

\footnotetext{
${ }^{17}$ It can be shown that counterterms are analytic in the lowenergy scales order by order in the EFT expansion.
}

would obtain using the EFT calculation of $\Pi\left(q^{2}\right)$ [which in this example we carried out to lowest order; i.e., we obtained an EFT representation of $\Pi(0)$, including the counterterm contribution $\Pi_{\mathrm{CT}}(0)$ ]. However, the integral over $q^{2}$ defining $a_{1}$ is itself divergent, and we thus will need to add new counterterms, which are only defined in the theory containing not only the pion, but also the photon and muon as dynamical fields, with the integral over $q^{2}$ representing the photon-muon loop. Of course, since in our model we have the exact expression (3.2), we know what $a_{2}$ is exactly, but we will see how it corresponds to counterterms in the pion-muon-photon EFT below.

A direct calculation of the first term in Eq. (3.11) gives

$$
a_{1}=m_{\mu}^{2}\left\{\Pi_{\mathrm{EFT}}(0)+\Pi_{\mathrm{CT}}(0)\right\}\left(-\frac{1}{\epsilon}-\log \frac{m_{\mu}^{2}}{\mu^{2}}\right),
$$


showing explicitly that this contribution is divergent; this divergence comes from the muon-photon loop in diagram T2. A direct calculation of the second term yields ${ }^{18}$

$$
\begin{aligned}
a_{2}= & m_{\mu}^{2}\left\{\Pi_{\mathrm{EFT}}(0)+\Pi_{\mathrm{CT}}(0)\right\}\left(\frac{1}{\epsilon}+\log \frac{m_{\sigma}^{2}}{\mu^{2}}\right) \\
& +\left(\frac{m_{\mu}^{2}}{16 \pi^{2} m_{\sigma}^{2}}\right)\left(-1+\frac{\pi^{2}}{3}\right),
\end{aligned}
$$

so that the total result is

$$
\begin{aligned}
a & =a_{1}+a_{2} \\
& =m_{\mu}^{2} \Pi(0) \log \frac{m_{\sigma}^{2}}{m_{\mu}^{2}}+\left(\frac{m_{\mu}^{2}}{16 \pi^{2} m_{\sigma}^{2}}\right)\left(-1+\frac{\pi^{2}}{3}\right) .
\end{aligned}
$$

Using Eq. (3.9) this equals the complete result, Eq. (3.5).

Equation (3.13) is the result in the underlying theory, which we do not know if we do not know the UV completion of the EFT. However, the divergence in $a_{1}$ demands that we add counterterms to the EFT that absorbs this divergence, yielding the contribution shown as the first term in Eq. (3.13). Again, the divergent and logarithmic terms in Eq. (3.13) can be inferred from those in Eq. (3.12) and dimensional analysis as the sum has to be finite and independent of $\mu$. The second term in Eq. (3.13) again corresponds to a finite contribution, which is unknown if we only have access to the EFT, and will thus be represented in the EFT by renormalized LECs.

In order to disentangle the complete counterterm structure, we split the different contributions as

$$
\begin{gathered}
a=\underbrace{m_{\mu}^{2} \Pi_{\mathrm{EFT}}(0)\left(-\frac{1}{\epsilon}-\log \frac{m_{\mu}^{2}}{\mu^{2}}\right)}_{(1)} \\
+\underbrace{m_{\mu}^{2} \Pi_{\mathrm{CT}}(0)\left(-\frac{1}{\epsilon}-\log \frac{m_{\mu}^{2}}{\mu^{2}}\right)}_{(2)} \\
+\underbrace{m_{\mu}^{2} \Pi_{\mathrm{EFT}}(0)\left(\frac{1}{\epsilon}+\log \frac{m_{\sigma}^{2}}{\mu^{2}}\right)}_{(3)} \\
+\underbrace{m_{\mu}^{2} \Pi_{\mathrm{CT}}(0)\left(\frac{1}{\epsilon}+\log \frac{m_{\sigma}^{2}}{\mu^{2}}\right)}_{(4)} \\
+\underbrace{\frac{m_{\mu}^{2}}{16 \pi^{2} m_{\sigma}^{2}}\left(-1+\frac{\pi^{2}}{3}\right)}_{(5)},
\end{gathered}
$$

\footnotetext{
${ }^{18}$ One way to do the calculation is to start from the first line of Eq. (3.4) for $\Pi\left(q^{2}\right)$, subtract $\Pi(0)$, carry out the integral over $q^{2}$ in Eq. (3.11), and finally the integral over $x$ in Eq. (3.4).
}

which may be expressed as

$$
\begin{gathered}
a=\underbrace{m_{\mu}^{2} \Pi_{\mathrm{EFT}}(0)\left(-\frac{1}{\epsilon}-\log \frac{m_{\mu}^{2}}{\mu^{2}}\right)}_{(1)} \\
+\underbrace{m_{\mu}^{2} C_{\psi_{\mu}^{2} A^{2}}(\mu)\left(-\frac{1}{\epsilon}-\log \frac{m_{\mu}^{2}}{\mu^{2}}\right)}_{(2)} \\
+\underbrace{m_{\mu}^{2} C_{\psi_{\mu}^{2} \pi^{2}}(\mu)\left(-\frac{2}{\epsilon}+\gamma_{E}+\log \frac{m_{\pi}^{2}}{4 \pi \mu^{2}}\right)}_{(3)} \\
+\underbrace{m_{\mu}^{2} C_{\psi_{\mu}^{2} A}(\mu)}_{(4)+(5)},
\end{gathered}
$$

where $^{19}$

$$
\begin{aligned}
C_{\psi_{\mu}^{2} A^{2}}(\mu) & =-\frac{1}{16 \pi^{2} m_{\sigma}^{2}}\left(\frac{2}{\epsilon}-\gamma_{E}-\log \frac{m_{\sigma}^{2}}{4 \pi \mu^{2}}+1\right), \\
C_{\psi_{\mu}^{2} \pi^{2}}(\mu) & =-\frac{1}{16 \pi^{2} m_{\sigma}^{2}}\left(\frac{1}{\epsilon}+\log \frac{m_{\sigma}^{2}}{\mu^{2}}\right), \\
C_{\psi_{\mu}^{2} A}(\mu)= & -\frac{1}{16 \pi^{2} m_{\sigma}^{2}}\left(\frac{2}{\epsilon}-\gamma_{E}-\log \frac{m_{\sigma}^{2}}{4 \pi \mu^{2}}+1\right)\left(\frac{1}{\epsilon}+\log \frac{m_{\sigma}^{2}}{\mu^{2}}\right) \\
& +\frac{1}{16 \pi^{2} m_{\sigma}^{2}}\left(-1+\frac{\pi^{2}}{3}\right) .
\end{aligned}
$$

Note the presence of terms proportional to $1 / \epsilon^{2}$, as expected at two loops, where the T4-type LEC $C_{\mu^{2} A^{2}}(\mu)$ already appeared in Eq. (3.10), and we encounter the new counterterms

$$
\begin{aligned}
& \text { T3: } \frac{C_{\psi_{\mu}^{2} \pi^{2}}(\mu)}{4} \psi_{\mu}^{2} \pi^{2}, \\
& \text { T5: } \frac{C_{\psi_{\mu}^{2} A}(\mu)}{2} \psi_{\mu}^{2} A .
\end{aligned}
$$

$a^{(1)}$ corresponds to the diagram T2 in Fig. 3. This diagram has two subdivergences, each needing a counterterm, one for the pion loop and one for the muon-photon loop, leading to counterterm vertices $\psi_{\mu}^{2} A^{2}$ and $\psi_{\mu}^{2} \pi^{2}$, respectively. The first vertex, $\psi_{\mu}^{2} A^{2}$, is obtained by contracting the pion loop to a point and was already encountered in the EFT calculation of $\Pi\left(q^{2}\right)$; it corresponds to diagram T4 and leads to $a^{(2)}$. The second vertex, $\psi_{\mu}^{2} \pi^{2}$, is new and is obtained by contracting the muon-photon loop to a point;

\footnotetext{
${ }^{19}$ In a minimal subtraction scheme, one drops the combinations $1 / \epsilon+$ finite constant to obtain the renormalized LECs.
} 
note that this counterterm involves both the pions of the "strong interactions" and the muons of "QED." This new counterterm corresponds to diagram T3 and leads to $a^{(3)}$. An overall two-loop divergence corresponding to the $1 / \epsilon^{2}$ pole requires a new counterterm proportional to $\psi_{\mu}^{2} A$. It corresponds to diagram $\mathrm{T} 5$ and is represented by the contribution $a^{(4)}$; this counterterm can also have (and does have, in the UV completion provided by our model) a finite part, $a^{(5)}$. This latter counterterm is to be compared to the Pauli term in the case of $a_{\mu}^{\mathrm{HVP}}$. The appearance of $1 / \epsilon^{2}$ poles is consistent with Eq. (3.2) being a two-loop integral. Again, the specific result for $a^{(5)}$ is only known because in this example we know the underlying theory; if we only had access to the EFT, the factor $-1+\pi^{2} / 3$ would be replaced by an unknown, finite constant. Similar calculations could be carried out to higher orders in $m_{\pi}^{2} / m_{\sigma}^{2}$ and $m_{\mu}^{2} / m_{\sigma}^{2}$.

\section{The toy model in finite volume}

We now turn to the analysis of the interplay between FV effects and UV divergences, our primary reason for introducing the toy model (3.1) in the first place. We begin with calculating FV effects in the model itself and then calculate and compare them with the calculation in the EFT developed in the previous subsection.

It is convenient to use the Schwinger parametrization for $\Pi\left(q^{2}\right)$ given by

$$
\begin{aligned}
\Pi\left(q^{2}\right)= & \int \frac{d^{4} p}{(2 \pi)^{4}} \int_{0}^{\infty} d \alpha_{1} \alpha_{1} \\
& \times \int_{0}^{\infty} d \alpha_{2} e^{-\alpha_{2}\left(p^{2}+m_{\sigma}^{2}\right)} e^{-\alpha_{1}\left((p-q)^{2}+m_{\pi}^{2}\right)} .
\end{aligned}
$$

Making the change of variables

$\hat{x}=\frac{\alpha_{1}}{\alpha_{1}+\alpha_{2}}, \quad x=\alpha_{1}+\alpha_{2} \leftrightarrow \alpha_{1}=x \hat{x}, \quad \alpha_{2}=x(1-\hat{x})$,

one can rewrite $\Pi\left(q^{2}\right)$ in a finite spatial volume with linear dimension $L$ and periodic boundary conditions as

$$
\begin{aligned}
\Pi_{\mathrm{FV}}\left(q_{4}^{2}\right)= & \sum_{\vec{n}} \Pi^{(n)}\left(q_{4}^{2}\right), \\
\Pi^{(n)}\left(q_{4}^{2}\right)= & \int_{0}^{1} d \hat{x} \hat{x} \int_{0}^{\infty} d x x^{2} e^{-x N^{2}} \int_{-\infty}^{\infty} \frac{d p}{2 \pi^{2}} p \\
& \times \int_{-\infty}^{\infty} \frac{d p_{4}}{2 \pi} e^{-x\left(p_{4}^{2}+p^{2}\right)} \frac{e^{i n p L}}{2 i n L},
\end{aligned}
$$

where

$$
\begin{aligned}
& N^{2}=q^{2} \hat{x}(1-\hat{x})+N_{0}^{2}, \\
& N_{0}^{2}=m_{\sigma}^{2}(1-\hat{x})+m_{\pi}^{2} \hat{x},
\end{aligned}
$$

and we made use of the Poisson resummation (2.23). As we are interested here in the FV contributions, we will take $n>0$. We took $\vec{q}=0$, as in Sec. II D and shifted $p_{4}-x q_{4} \rightarrow p_{4}$, and $q^{2}=q_{4}^{2}>0$. Carrying out the integrals over $p_{4}$ and $p$ yields

$\Pi^{(n)}\left(q^{2}\right)=\frac{1}{16 \pi^{2}} \int_{0}^{1} d \hat{x} \hat{x} \int_{0}^{\infty} d x e^{-x q^{2} \hat{x}(1-\hat{x})} e^{-x N_{0}^{2}} e^{-n^{2} L^{2} /(4 x)}$.

We can also write

$$
a_{\mathrm{FV}}=\sum_{\vec{n}} a^{(n)},
$$

so that, performing the integrals over $q^{2}$ in Eq. (3.2) and $x$ in Eq. (3.23), we find

$$
\begin{aligned}
a^{(n)}= & -\frac{m_{\mu}^{2}}{16 \pi^{2}} \int_{0}^{1} d \hat{x} \frac{\hat{x}}{N_{0}^{2}}\left(K_{0}\left(N_{0} n L\right)\right. \\
& \left.+N_{0} n L K_{1}\left(N_{0} n L\right)\left(\gamma_{E}+\log \frac{m_{\mu}^{2} n^{2} L^{2} \hat{x}(1-\hat{x})}{2 N_{0} n L}\right)\right),
\end{aligned}
$$

where $K_{\nu}(z)$ is the modified Bessel function of order $\nu$. Carrying out the integral over $\hat{x}$ (see Appendix B for details), we find for our final result

$$
a^{(n)}=m_{\mu}^{2} \underbrace{\frac{1}{8 \pi^{2}} \frac{1}{m_{\sigma}^{2}} K_{0}\left(m_{\pi} n L\right)}_{\Pi_{\mathrm{EFT}}^{(n)}(0)} \log \frac{m_{\sigma}^{2}}{m_{\mu}^{2}} .
$$

Here we dropped terms $\sim e^{-m_{\sigma} n L}$ and terms that are suppressed by additional powers of $m_{\pi}^{2} / m_{\sigma}^{2}$ or $1 /\left(m_{\sigma}^{2} L^{2}\right)$. In Eq. (3.26), we already anticipate the result to be derived in Eq. (3.27) below, that the prefactor of the logarithm is nothing but $m_{\mu}^{2} \Pi_{\mathrm{EFT}}^{(n)}(0)$ in finite volume. This result conforms with the intuition that FV effects associated with the pions are infrared effects due to the low-energy degrees of freedom contained in the EFT. As the $\sigma$ is not part of the EFT, its FV effects cannot be obtained from the EFT.

We now turn to the calculation of FV effects in the EFT version of our toy model, beginning with $\Pi_{\mathrm{EFT}}(0)$. Defining $\Pi_{\mathrm{EFT}}^{(n)}$ analogous to the definition of $D^{(n)}$ in Eq. (2.24), we obtain for $n>0$

$$
\begin{aligned}
\Pi_{\mathrm{EFT}}^{(n)}(0) & =\frac{1}{m_{\sigma}^{2}} \int_{-\infty}^{\infty} \frac{d p_{4}}{2 \pi} \int_{-\infty}^{\infty} \frac{d p}{2 \pi^{2}} \frac{p}{\left(p_{4}^{2}+p^{2}+m_{\pi}^{2}\right)^{2}} \frac{e^{i p n L}}{2 i n L} \\
& =\frac{1}{8 \pi^{2}} \frac{1}{m_{\sigma}^{2}} K_{0}\left(m_{\pi} n L\right) \\
& =\frac{1}{8 \pi^{2}} \frac{1}{m_{\sigma}^{2}} \sqrt{\frac{\pi}{2}} \frac{e^{-m_{\pi} n L}}{\sqrt{m_{\pi} n L}}\left(1+\mathcal{O}\left(\frac{1}{m_{\pi} n L}\right)\right),
\end{aligned}
$$


where we evaluated the integral over $p$ using the residue theorem and then the integral over $p_{4}$ to yield the modified Bessel function.

There is another instructive way to obtain the result in Eq. (3.27). This is by noting that the case $n>0$ is equivalent to putting the particle propagating in the loop on shell. Going to Minkowski space, and after using Eq. (2.23) again, one has

$$
\begin{aligned}
\Pi_{\mathrm{EFT}}^{(n)}(0)= & \left(\frac{-i}{m_{\sigma}^{2}}\right) \int_{-\infty}^{\infty} \frac{d p_{0}}{2 \pi} \\
& \times \int_{-\infty}^{\infty} \frac{d p}{2 \pi^{2}} \frac{p}{\left(p_{0}^{2}-p^{2}-m_{\pi}^{2}+i \epsilon\right)^{2}} \frac{e^{i n p L}}{2 i n L} .
\end{aligned}
$$

Putting the pion in the loop on shell amounts to the following replacement:

$$
\begin{aligned}
\frac{1}{\left(p_{0}^{2}-p^{2}-m_{\pi}^{2}+i \epsilon\right)^{2}} & =\frac{1}{2 p} \frac{d}{d p}\left(\frac{1}{p_{0}^{2}-p^{2}-m_{\pi}^{2}+i \epsilon}\right) \\
& \rightarrow-\frac{i \pi}{2 p} \frac{d}{d p} \delta\left(-p_{0}^{2}+p^{2}+m_{\pi}^{2}\right),
\end{aligned}
$$

so that Eq. (3.28) becomes

$$
\begin{aligned}
& \Pi_{\mathrm{EFT}}^{(n)}(0) \\
& \quad=\frac{-1}{m_{\sigma}^{2}}\left(\frac{1}{8 \pi^{2}}\right) \int_{-\infty}^{\infty} d p_{0} \int_{-\infty}^{\infty} d p \frac{d}{d p}\left[\delta\left(p_{0}^{2}-p^{2}-m_{\pi}^{2}\right)\right] \frac{e^{i n p L}}{2 i n L} \\
& =\frac{1}{m_{\sigma}^{2}} \frac{1}{8 \pi^{2}} \int_{m_{\pi}^{2}}^{\infty} \frac{d p_{0}}{\sqrt{p_{0}^{2}-m_{\pi}^{2}}} \cos \left(n L \sqrt{p_{0}^{2}-m_{\pi}^{2}}\right)
\end{aligned}
$$

A change of variable $y=\sqrt{p_{0}^{2}-m_{\pi}^{2}}$ finally yields

$$
\Pi_{\mathrm{EFT}}^{(n)}(0)=\frac{1}{m_{\sigma}^{2}} \frac{1}{16 \pi^{2}} \int_{-\infty}^{\infty} \frac{d y}{\sqrt{y^{2}+m_{\pi}^{2}}} e^{i n L y},
$$

which equals Eq. (3.27). We note that the result for $\Pi_{\mathrm{EFT}}^{(n)}(0)$ for $n>0$ is finite; the counterterm $\Pi_{\mathrm{CT}}(0)$ was only needed in the infinite-volume theory. It does not involve a pion loop, so there is no contribution to the FV part of $\Pi(0)$ in the EFT. In other words, $\Pi_{\mathrm{CT}}(0)$ renormalizes $\Pi_{\mathrm{EFT}}(0)$ in infinite volume, while FV corrections to $\Pi_{\mathrm{EFT}}(0)$ are finite.

The implication of this is that we expect the FV part of $\Pi_{\mathrm{CT}}(0)$ to vanish. Since we calculated $\Pi_{\mathrm{EFT}}^{(n)}(0)$ to order $1 / m_{\sigma}^{2}$ we thus expect that if we calculate $\Pi_{\mathrm{CT}}^{(n)}(0)$ for $n>0$ in the same way that we calculated $\Pi_{\mathrm{CT}}(0)$ from the model in Eq. (3.8) it will turn out not to have a term of order $1 / m_{\sigma}^{2}$, implying that the FV corrections to Eq. (3.8) vanish. This is what we will demonstrate next.

Replacing the $\sigma$ propagator in Eq. (3.3) by the second term of Eq. (3.6), and again going through the steps to arrive at an expression for the $\mathrm{FV}$ correction term $\Pi_{\mathrm{CT}}^{(n)}(0)$ for $n>0$, we arrive at

$$
\begin{aligned}
\Pi_{\mathrm{CT}}^{(n)}(0)= & -\frac{1}{m_{\sigma}^{2}} \int_{-\infty}^{\infty} \frac{d p_{4}}{2 \pi} \int_{-\infty}^{\infty} \frac{d p}{2 \pi^{2}} \frac{p}{\left(p_{4}^{2}+p^{2}+m_{\pi}^{2}\right)^{2}} \\
& \times\left[\frac{p_{4}^{2}+p^{2}}{p_{4}^{2}+p^{2}+m_{\sigma}^{2}}\right] \frac{e^{i p n L}}{2 i n L} .
\end{aligned}
$$

In addition to the double poles at $p= \pm i \sqrt{p_{4}^{2}+m_{\pi}^{2}}$ also present in Eq. (3.27), the integrand now also has simple poles at $p= \pm i \sqrt{p_{4}^{2}+m_{\sigma}^{2}}$. The pole at $p=i \sqrt{p_{4}^{2}+m_{\sigma}^{2}}$ gives a contribution $\sim e^{-L \sqrt{p_{4}^{2}+m_{\sigma}^{2}}}$ which, however, is negligible when $m_{\sigma} \gg m_{\pi}$, in comparison to the contribution $\sim e^{-L \sqrt{p_{4}^{2}+m_{\pi}^{2}}}$ from the pole at $p=i \sqrt{p_{4}^{2}+m_{\pi}^{2}}$, and may thus be discarded. ${ }^{20}$ Furthermore, we can immediately see why the remaining part of $\Pi_{\mathrm{CT}}^{(n)}(0)$ is suppressed relative to $\Pi_{\mathrm{EFT}}^{(n)}(0)$ by an extra factor $m_{\pi}^{2} / m_{\sigma}^{2}$. The suppression by this factor follows from the presence of the term in square brackets in Eq. (3.32) and dimensional analysis. The factor $e^{-L \sqrt{p_{4}^{2}+m_{\pi}^{2}}}$ present after integration over $p$ makes the integral over $p_{4}$ sufficiently convergent that the rest of the integrand can be expanded in inverse powers of $m_{\sigma}^{2}$. We conclude that, to the order we are working, indeed, no counterterm FV correction is produced by the underlying model. We note that, in this argument, we always assume that $m_{\pi} L \gg 1$, i.e., that we consider FV effects in the $p$ regime.

To summarize, to leading order in an expansion in $m_{\pi}^{2} / m_{\sigma}^{2}$, we conclude that, for $n>0$,

$$
\Pi_{\mathrm{CT}}^{(n)}(0)=0 \quad \Rightarrow \quad \Pi^{(n)}(0)=\Pi_{\mathrm{EFT}}^{(n)}(0) .
$$

No counterterm is needed for the FV corrections to $\Pi(0)$ calculated using the EFT.

Now, let us return to our "anomaly," $a$ defined in Eq. (3.2). First, substituting Eq. (3.27) into Eq. (3.2) one finds, for the finite-volume part of $a$ in the EFT,

$$
\begin{aligned}
a_{\mathrm{EFT}}^{(n)} & =\int_{0}^{\infty} d q^{2}\left(\frac{q^{2}}{\mu^{2}}\right)^{\epsilon} \frac{m_{\mu}^{2}}{q^{2}+m_{\mu}^{2}} \Pi_{\mathrm{EFT}}^{(n)}(0) \\
& =m_{\mu}^{2} \Pi_{\mathrm{EFT}}^{(n)}(0)\left(-\frac{1}{\epsilon}-\log m_{\mu}^{2}\right) \\
& =m_{\mu}^{2} \Pi^{(n)}(0)\left(-\frac{1}{\epsilon}-\log \frac{m_{\mu}^{2}}{\mu^{2}}\right)
\end{aligned}
$$

This corresponds to the FV part of $a_{1}$ defined in Eq. (3.11): it is the FV contribution from the pion loop in diagram T2 in Fig. 3. Clearly, a counterterm contribution will be needed

\footnotetext{
${ }^{20}$ This reflects the fact that light particles dominate the FV effects in the EFT for our toy model.
} 
to make this FV contribution finite. The counterterm needed here is the same infinite-volume counterterm used in diagram T3 of Fig. 3, with the pion loop in that diagram producing the FV part. Diagrams T4 and T5 do not contribute to the pion-induced FV corrections (T4 would only yield FV contributions due to a muon wrapping around the world). We emphasize how it is consistent to keep the $q^{2}$ integral in Eq. (3.11) in infinite volume, even though $\Pi(0)$ itself is replaced by its $\mathrm{FV}$ part. In other words, we can treat the "strong-interaction" (i.e., $\pi, \sigma$ ) physics in finite volume, while keeping the "electromagnetic" (i.e., $A, \mu$ ) physics in infinite volume.

Finally, as before, we need to consider $a_{2}$ given by [cf. Eq. (3.11)]

$$
a_{2}=m_{\mu}^{2} \int_{0}^{\infty} \frac{d q^{2}}{q^{2}+\underbrace{m_{\mu}^{2}}_{\text {neglect }}}\left(\frac{q^{2}}{\mu^{2}}\right)^{\epsilon}\left\{\Pi\left(q^{2}\right)-\Pi(0)\right\},
$$

but now replacing the pion-physics part $\Pi\left(Q^{2}\right)-\Pi(0)$ by its FV correction. As before, the $m_{\mu}$ in the denominator can be neglected because $\Pi\left(q^{2}\right)-\Pi(0) \sim q^{2}$ as $q^{2} \rightarrow 0$. The term proportional to $\Pi(0)$ in Eq. (3.35) then vanishes in dimensional regularization, so the net result becomes

$$
a_{2}^{(n)}=m_{\mu}^{2} \int_{0}^{\infty} \frac{d q^{2}}{q^{2}}\left(\frac{q^{2}}{\mu^{2}}\right)^{\epsilon} \Pi^{(n)}\left(q^{2}\right) .
$$

Inserting Eq. (3.23) and using the method of Appendix B that was used to evaluate Eq. $(3.25),{ }^{21}$ we obtain

$$
a_{2}^{(n)}=m_{\mu}^{2} \Pi^{(n)}(0)\left(\frac{1}{\epsilon}+\log \frac{m_{\sigma}^{2}}{\mu^{2}}\right) .
$$

An important remark here is that, even though the end result (3.37) is proportional to $\Pi^{(n)}(0)$, the $q^{2}$ dependence of $\Pi^{(n)}\left(q^{2}\right)$ in the integrand in Eq. (3.36) is crucial for obtaining this result. The factor $1 / \epsilon+\log \left(m_{\sigma}^{2} / \mu^{2}\right)$ is the same as that appearing in $a^{(3)}$ in infinite volume, on the third and fourth lines of Eq. (3.15). Thus, adding Eqs. (3.34) to (3.37), one arrives at

$$
a^{(n)}=a_{1}^{(n)}+a_{2}^{(n)}=m_{\mu}^{2} \Pi^{(n)}(0) \log \frac{m_{\sigma}^{2}}{m_{\mu}^{2}},
$$

where $\Pi^{(n)}(0)=\Pi_{\mathrm{EFT}}^{(n)}(0)$ and $\Pi_{\mathrm{EFT}}^{(n)}(0)$ is given in Eq. (3.27). This is equal to the complete result (3.26) calculated in the underlying toy model. In summary, the volume dependence is contained in the contribution from the EFT, i.e., $\Pi^{(n)}(0)$, but the infinite-volume counterterm with coefficient $C_{\psi_{\mu}^{2} \pi^{2}}(\mu)$ given in Eq. (3.18b) is needed to

\footnotetext{
${ }^{21}$ For the $1 / \epsilon$ part, all that is needed is $K_{1}(y)=-d K_{0}(y) / d y$.
}

render the FV contribution finite. The contributions $a^{(4)}$ and $a^{(5)}$ in Eq. (3.15) do not contribute pion-induced FV effects, as the corresponding diagrams T4 and T5 in Fig. 3 do not contain pion loops.

\section{CONCLUSION}

In this paper, we considered the effective field theory approach to the hadronic vacuum polarization contribution to the muon anomalous magnetic moment, $a_{\mu}^{\mathrm{HVP}}$. Our primary aim was a better understanding of how the evaluation of finite-volume effects works in an EFT framework, but this led us to a discussion of the counterterm structure needed for a complete EFT representation of $a_{\mu}^{\mathrm{HVP}}$ in infinite volume. The specific motivation for our interest in finite-volume effects is to contribute to a deeper understanding of the systematic error caused by these effects in lattice computations of $a_{\mu}^{\mathrm{HVP}}$.

Finite-volume effects in lattice computations of $a_{\mu}^{\mathrm{HVP}}$ are dominated by pions, and thus the natural EFT candidate is chiral perturbation theory. As we showed, for a complete low-energy description of $a_{\mu}^{\mathrm{HVP}}$, also muons and photons have to be included in the EFT. In particular, since $a_{\mu}^{\mathrm{HVP}}$ also contains a loop with muons and photons, counterterms in our EFT can contain not only pion fields, but also muon and photon fields. Just like counterterms are needed to regulate the UV behavior of pion loops in ChPT, additional counterterms are also needed to regulate the UV behavior of the muon-photon loop. These counterterms lead to the presence of new low-energy constants in the EFT, in addition to those already present in ChPT. The values of these LECs can, of course, only be fixed by matching with the underlying UV-complete theory, QCD plus QED.

Once these LECs are taken into account, ChPT, augmented with muon and photon fields, gives a complete representation of $a_{\mu}^{\mathrm{HVP}}$. In addition, finite-volume effects at any given order are completely fixed in terms of the LECs that appear to one order less in the EFT. In this sense, finitevolume effects can be predicted, at any given order, in terms of the LECs of the infinite-volume EFT. This is not surprising, as $a_{\mu}^{\mathrm{HVP}}$ is nothing else than (the projection to zero external photon momentum and onto the Pauli spin structure of) a correlation function in QCD plus QED.

In the case of $a_{\mu}^{\mathrm{HVP}}$, we explained that the need for new counterterms, not present in ChPT, the EFT for pions alone, arises for the first time in the EFT expansion at $\mathrm{N}^{3} \mathrm{LO}$ and that their role in the study of finite-volume effects starts at $\mathrm{N}^{4} \mathrm{LO}$. We constructed the new counterterms at the lowest order at which they appear and showed that indeed dimensional arguments imply that they become relevant at $\mathrm{N}^{3} \mathrm{LO}$. While the construction of these counterterms is relatively straightforward, it would require three- and four-loop calculations to demonstrate how it all works in the case of $a_{\mu}^{\mathrm{HVP}}$. Therefore, instead, we demonstrated our observations by working out a toy model in which the effects 
appear already at two loops, which is the minimum number. While it is unlikely that the full $\mathrm{N}^{3} \mathrm{LO}$ analysis will ever be worked out in practice, it is important to establish the validity of the EFT framework for the study of $a_{\mu}^{\mathrm{HVP}}$ in order to be assured that even the NNLO analysis of finitevolume effects carried out in Ref. [10] has a solid EFT basis. We believe that our discussion in this paper illustrates why indeed this is the case. We expect the same separation between the UV physics represented by the counterterms and IR physics of finite-volume effects will also take place in the hadronic light-by-light contribution when the QED part is taken in infinite volume [22].

\section{ACKNOWLEDGMENTS}

We thank Max Hansen for discussions. T. B.'s and M. G.'s work is supported by the U.S. Department of Energy, Office of Science, Office of High Energy Physics, under Grants No. DE-SC0010339 and No. DESC0013682, respectively. S.P. is supported by CICYTFEDER-FPA2017-86989-P and by Grant No. 2017 SGR 1069.

\section{APPENDIX A: COUNTERTERMS IN THE TIME-MOMENTUM REPRESENTATION}

In this Appendix, we revisit the time-momentum representation for the HVP [20]. In lattice computations, $a_{\mu}^{\mathrm{HVP}}$ is often obtained from

$$
C(t)=\frac{1}{3} \sum_{i} \int d^{3} x\left\langle j_{i}(0,0) j_{i}(\vec{x}, t)\right\rangle,
$$

where $j_{\mu}$ is the hadronic part of the electromagnetic current. We can, of course, express $C(t)$ in terms of the HVP $\Pi\left(q^{2}\right)$ :

$$
\begin{aligned}
C(t) & =\frac{1}{3} \sum_{i} \int d^{3} x \int \frac{d^{4} q}{(2 \pi)^{4}} e^{i q x}\left(\delta_{i i} q^{2}-q_{i} q_{i}\right) \Pi\left(q^{2}\right) \\
& =\int \frac{d q_{4}}{2 \pi} e^{i q_{4} t} q_{4}^{2} \Pi\left(q_{4}^{2}\right) \\
& =-\int \frac{d q_{4}}{2 \pi} e^{i q_{4} t} q_{4}^{2} \hat{\Pi}\left(q_{4}^{2}\right)-\Pi(0) \delta^{\prime \prime}(t),
\end{aligned}
$$

where

$$
\hat{\Pi}\left(q^{2}\right)=\Pi(0)-\Pi\left(q^{2}\right) .
$$

Equation (A2) contains the divergent counterterm $-\Pi(0) \delta^{\prime \prime}(t)$, and we note that, in general, counterterms in $C(t)$ live at $t=0$ and thus appear in the form of the Dirac delta function $\delta(t)$ and its derivatives. In particular, in ChPT, higher derivatives than $\delta^{\prime \prime}(t)$ can appear, corresponding to the fact that counterterms are polynomials in $q^{2}$ in the momentum representation.
Inverting Eq. (A2), we find

$$
\begin{aligned}
q_{4}^{2} \Pi\left(q_{4}^{2}\right) & =\int_{-\infty}^{\infty} d t\left(e^{-i q_{4} t}-1\right) C(t) \\
& =-4 \int_{0}^{\infty} d t \sin ^{2}\left(q_{4} t / 2\right) C(t),
\end{aligned}
$$

where we inserted the -1 to enforce that the left-hand side vanishes for $q_{4}=0$ and we used that $C(t)$ is even in $t$ in the second step. It follows that

$$
\hat{\Pi}\left(q_{4}^{2}\right)=\int_{0}^{\infty} d t\left(\frac{4 \sin ^{2}\left(q_{4} t / 2\right)}{q_{4}^{2}}-t^{2}\right) C(t),
$$

and thus

$$
\begin{aligned}
a_{\mu}^{\mathrm{HVP}} & =\int_{0}^{\infty} d q^{2} f\left(q^{2}\right) \hat{\Pi}\left(q^{2}\right) \\
& =\int_{0}^{\infty} d q^{2} f\left(q^{2}\right) \int_{0}^{\infty} d t\left(\frac{4 \sin ^{2}(q t / 2)}{q^{2}}-t^{2}\right) C(t) .
\end{aligned}
$$

In Ref. [10] we made the incorrect observation that at NNLO $a_{\mu}^{\mathrm{HVP}}$ is UV divergent in ChPT, because at NNLO $C(t) \sim 1 / t^{5}$ for $t \rightarrow 0$, which makes the integral over $t$ in Eq. (A6) divergent, as the weight function in the integral over $t$ behaves like $t^{4}$ for $t \rightarrow 0$. If true, this would be a paradox, because, as we have seen in Sec. I, the expression for $a_{\mu}^{\mathrm{HVP}}$ in terms of $\hat{\Pi}\left(q^{2}\right)$ is UV finite at NNLO in ChPT. The problem, in fact, has nothing to do with $a_{\mu}^{\mathrm{HVP}}$, as can be seen from Eq. (A5). While $\hat{\Pi}\left(q^{2}\right)$ is finite by construction, the integral on the right-hand side of this equation is UV divergent if indeed $C(t) \sim 1 / t^{5}$ for small $t$ at NNLO.

As we will show here, this apparent paradox arises if one is not careful with the treatment of counterterms in the timemomentum representation. The correct expression connecting $\hat{\Pi}\left(q^{2}\right)$ and $C(t)$ in ChPT is

$$
\begin{aligned}
\hat{\Pi}\left(q^{2}\right)= & \lim _{\epsilon \rightarrow 0} \int_{0^{-}}^{\infty} d t\left(\frac{4 \sin ^{2}(q t / 2)}{w^{2}}-t^{2}\right) \\
& \times\left(C_{\mathrm{ChPT}}(t ; \epsilon, \mu)-\frac{1}{2} \delta^{\mathrm{IV}}(t) C(\epsilon, \mu)\right),
\end{aligned}
$$

where $\delta^{\mathrm{IV}}(t)$ is the fourth derivative of the Dirac delta and $C(\epsilon, \mu)$ is a counterterm directly related to the LECs of ChPT. As usual, in order to define a counterterm, a regulator needs to be introduced, and the parameter $\epsilon$ represents this regulator, as we will see below. The rest of this Appendix will prove this result. While we keep our discussion concrete by using a simple example to make the argument, the end result carries over to the case of ChPT. 
In the following, it will be useful to express $C(t)$ in terms of the spectral function $\rho(s)$. From the subtracted dispersion relation

$$
\hat{\Pi}\left(q^{2}\right)=q^{2} \int_{0}^{\infty} d s \frac{\rho(s)}{s\left(s+q^{2}\right)},
$$

it follows from Eq. (A2) that

$$
\begin{aligned}
C(t)= & -\int_{0}^{\infty} d s \rho(s) \int_{-\infty}^{\infty} \frac{d q_{4}}{2 \pi} e^{i q_{4} t} \frac{q_{4}^{4}}{s\left(s+q_{4}^{2}\right)}-\Pi(0) \delta^{\prime \prime}(t) \\
= & -\int_{0}^{\infty} d s \rho(s)\left(-\delta(t)-\frac{1}{s} \delta^{\prime \prime}(t)+\frac{1}{2} \sqrt{s} e^{-\sqrt{s}|t|}\right) \\
& -\Pi(0) \delta^{\prime \prime}(t) \\
= & -\int_{0}^{\infty} d s \rho(s)\left(-\delta(t)+\frac{1}{2} \sqrt{s} e^{-\sqrt{s}|t|}\right),
\end{aligned}
$$

where in the last step we used the unsubtracted dispersion relation.

To illustrate the claim and understand what is going on, let us consider the simple example of a spectral function given by

$$
\rho(s)=\left(1+\frac{s}{s+M^{2}}\right) \theta\left(s-m^{2}\right),
$$

where $M$ is like the $\rho$-meson mass, for example, and we are interested in a "ChPT" result where $M^{2} \gg m^{2} \sim q^{2} \equiv m_{L}^{2}$ ( $L$ for light). With this spectral function, one may calculate the once-subtracted vacuum polarization $\hat{\Pi}\left(Q^{2}\right)$ as

$$
\begin{aligned}
\hat{\Pi}\left(q^{2}\right) & =q^{2} \int_{m^{2}}^{\infty} \frac{d s}{s\left(s+q^{2}\right)} \rho(s) \\
& =\log \frac{m^{2}+q^{2}}{m^{2}}+\frac{q^{2}}{M^{2}-q^{2}} \log \frac{m^{2}+M^{2}}{m^{2}+q^{2}} \\
& \approx \log \frac{m^{2}+q^{2}}{m^{2}}+\frac{q^{2}}{M^{2}} \log \frac{M^{2}}{m^{2}+q^{2}}+\mathcal{O}\left(\frac{m_{L}^{4}}{M^{4}}\right) .
\end{aligned}
$$

In this world, the identification of the counterterm can be made by splitting the spectral function as

$$
\rho(s)=\underbrace{1+\frac{s}{M^{2}}}_{\rho_{\mathrm{ChPT}}(s)}-\underbrace{\frac{s^{2}}{M^{2}\left(s+M^{2}\right)}}_{\Delta \rho(s)}
$$

in terms of the "ChPT" spectral function $\rho_{\mathrm{ChPT}}(s)$ and the "UV completion" $\Delta \rho(s)$. With this split, also the dispersion relation (A11) splits into two parts:

$$
\begin{aligned}
\hat{\Pi}\left(q^{2}\right)= & q^{2} \int_{m^{2}}^{\infty} \frac{d s}{s\left(s+q^{2}\right)}\left(1+\frac{s}{M^{2}}\right)\left(\frac{s}{\mu^{2}}\right)^{\epsilon} \\
& +q^{2} \int_{\underbrace{m^{2}}_{0}}^{\infty} \frac{d s}{s(s+\underbrace{q^{2}}_{0})}\left(-\frac{s^{2}}{M^{2}\left(s+M^{2}\right)}\right)\left(\frac{s}{\mu^{2}}\right)^{\epsilon} \\
\equiv & \hat{\Pi}_{\mathrm{ChPT}}\left(q^{2}, \epsilon, \mu\right)+q^{2} C(\epsilon, \mu),
\end{aligned}
$$

where

$\hat{\Pi}_{\mathrm{ChPT}}\left(q^{2}, \epsilon, \mu\right)=q^{2} \int_{m^{2}}^{\infty} \frac{d s}{s\left(s+q^{2}\right)}\left(1+\frac{s}{M^{2}}\right)\left(\frac{s}{\mu^{2}}\right)^{\epsilon}$

and

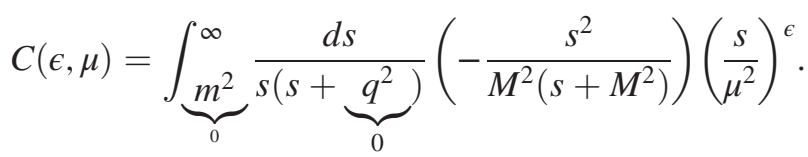

We have added a regulating factor $\left(q^{2} / \mu^{2}\right)^{\epsilon}$ because both integrals are now separately UV divergent. In the second integral, Eq. (A15), we may take the limit $q^{2}, m^{2} \rightarrow 0$ as it is IR convergent thanks to the subtraction $\Delta \rho(s)$, making $C(\epsilon, \mu)$ depend only on the UV scale $M$. Both integrals may be evaluated to give

$$
\begin{aligned}
\hat{\Pi}\left(q^{2}\right)= & \left(1-\frac{q^{2}}{M^{2}}\right) \log \left(1+\frac{q^{2}}{m^{2}}\right)-\frac{q^{2}}{M^{2}}\left(\frac{1}{\epsilon}+\log \frac{m^{2}}{\mu^{2}}\right) \\
& +q^{2} C(\epsilon, \mu)
\end{aligned}
$$

and

$$
C(\epsilon, \mu)=\frac{1}{M^{2}}\left(\frac{1}{\epsilon}+\log \frac{M^{2}}{\mu^{2}}\right) .
$$

$C(\epsilon, \mu)$ is the necessary counterterm and encodes the UV completion of the theory. One can check that the combined result (A16) agrees with the last line of Eq. (A11), as it should.

Let us now discuss consider Eq. (A7), using our example. Using Eqs. (A9) and (A12),

$$
\begin{aligned}
C_{\mathrm{ChPT}}(t)= & -\int_{m^{2}}^{\infty} d s \rho_{\mathrm{ChPT}}(s)\left(\frac{\sqrt{s}}{2} e^{-t \sqrt{s}}-\delta(t)\right)\left(t>0^{-}\right) \\
= & \frac{e^{-m t}}{t^{5}}\left[t^{2}(-2-m t(2+m t))\right. \\
& \left.+\frac{-24-m t(24+m t(12+m t(4+m t)))}{M^{2}}\right] \\
\approx & -\frac{2}{t^{3}}-\frac{24}{M^{2} t^{5}}\left(t \rightarrow 0^{+}\right) .
\end{aligned}
$$


The term proportional to $\delta(t)$ will not contribute to $\hat{\Pi}\left(q^{2}\right)$ in Eq. (A7), since the kernel $\left(\frac{4 \sin ^{2}(w t / 2)}{w^{2}}-t^{2}\right) \sim t^{4}$, and we will thus neglect this term in what follows. The result in Eq. (A18) shows that our example (A10) reproduces the $1 / t^{5}$ behavior for $t \rightarrow 0$ of ChPT at NNLO in the real world and, consequently, it also makes $\hat{\Pi}\left(q^{2}\right)$ in Eq. (A5) diverge. In view of Eq. (A11), this result is clearly incorrect.

First, using for instance Eq. (A14), we need to regulate this divergence. ${ }^{22}$ The result of Eq. (A14) may be expressed as

$\hat{\Pi}_{\mathrm{ChPT}}\left(q^{2}, \epsilon, \mu\right)=\int_{0}^{\infty} d t\left(\frac{4 \sin ^{2}(q t / 2)}{q^{2}}-t^{2}\right) C_{\mathrm{ChPT}}(t ; \epsilon, \mu)$,

with

$$
C_{\mathrm{ChPT}}(t ; \epsilon, \mu)=-\int_{m^{2}}^{\infty} d s\left(1+\frac{s}{M^{2}}\right) \frac{\sqrt{s}}{2} e^{-t \sqrt{s}}\left(\frac{s}{\mu^{2}}\right)^{\epsilon} .
$$

Then, using the identity

$$
q^{2}=\int_{0^{-}}^{\infty} d t\left(\frac{4 \sin ^{2}(q t / 2)}{q^{2}}-t^{2}\right)\left(-\frac{1}{2} \delta^{\mathrm{IV}}(t)\right),
$$

we can express the result in Eq. (A16) as

$$
\begin{aligned}
\hat{\Pi}\left(q^{2}\right)= & \int_{0^{-}}^{\infty} d t\left(\frac{4 \sin ^{2}(q t / 2)}{q^{2}}-t^{2}\right) \\
& \times\left(C_{\mathrm{ChPT}}(t ; \epsilon, \mu)-\frac{1}{2} \delta^{\mathrm{IV}}(t) C(\epsilon, \mu)\right),
\end{aligned}
$$

which is precisely Eq. (A7), as promised. To verify this, it is easiest to do the $t$ integral first, then express the integral over $s$ in terms of the Beta function, and finally, to use the identities $(x>0$ and $\epsilon<0)$ :

$$
\begin{aligned}
(-1)^{1+\epsilon} B(-x,-\epsilon, 0) & =\frac{1}{\epsilon}+\log \frac{1+x}{x}+\mathcal{O}(\epsilon), \\
(-1)^{1+\epsilon} B(-x, 1-\epsilon, 0) & =\log (1+x)+\mathcal{O}(\epsilon) .
\end{aligned}
$$

Our example generalizes to ChPT, because it shares the $1 / t^{5}$ behavior at NNLO. In order to properly treat the divergence arising in Eq. (A7), $C_{\mathrm{ChPT}}(t)$ needs to be properly regulated, and thus a counterterm needs to be introduced to define the integral when the regulator is removed. In momentum space, the counterterm is

\footnotetext{
${ }^{22}$ Alternatively one could also introduce a $t^{\eta}$ for $\eta \rightarrow 0$ in the integrand of Eq. (A18) as a regulator. The difference will be a redefinition of the counterterm $C(\epsilon, \mu)$ in Eq. (A17) but the final result for $\hat{\Pi}\left(q^{2}\right)$ will be the same.
}

proportional to $q^{2}$, as follows from Eq. (A21), and it corresponds to the LEC $c_{56}$ in two-flavor ChPT [21].

\section{APPENDIX B: INTEGRALS}

In this Appendix, we provide the details of some calculations in the main text. We begin with a derivation of the explicit form of the third line of Eq. (2.19). Starting from Eq. (2.16b), the additional term containing $m_{\pi}^{2}$ can be written as

$$
\delta a_{\mathrm{CT}}=\frac{m^{4}}{f_{\pi}^{4}} \int_{0}^{1} d x \int_{0}^{\infty} \frac{d q^{2}}{q^{2}+M^{2}}\left(q^{2}\right)^{\epsilon} \log \frac{q^{2} x(1-x)+m_{\pi}^{2}}{q^{2} x(1-x)},
$$

which is finite so that the limit $\epsilon \rightarrow 0$ may be taken. One notices that the "heavy propagator" admits a Mellin-Barnes representation

$$
\begin{aligned}
\frac{1}{q^{2}+M^{2}} & =\frac{1}{M^{2}} \sum_{n=0}^{\infty}(-1)^{n}\left(\frac{q^{2}}{M^{2}}\right)^{n} \\
& =\frac{1}{M^{2}} \frac{1}{2 \pi i} \int_{\gamma} d s\left(\frac{q^{2}}{M^{2}}\right)^{-s} \pi \csc (\pi s), \quad 0<\operatorname{Re} s<1,
\end{aligned}
$$

where $\gamma$ is a line parallel to the imaginary $s$ axis with $0<\operatorname{Re} s<1$. [One can see this by using

$$
\pi \csc (\pi s)=\sum_{n=-\infty}^{\infty} \frac{(-1)^{n}}{n+s}
$$

and then closing the contour in the left half plane counterclockwise.] Then,

$$
\begin{aligned}
\delta a_{\mathrm{CT}}= & \frac{m^{4}}{f_{\pi}^{4}} \frac{1}{M^{2}} \frac{1}{2 \pi i} \int_{\gamma} d s \pi \csc (\pi s) \int_{0}^{1} d x \int_{0}^{\infty} d q^{2}\left(\frac{q^{2}}{M^{2}}\right)^{-s} \\
& \times \log \frac{q^{2} x(1-x)+m^{2}}{q^{2} x(1-x)} \\
= & \frac{m^{4}}{f_{\pi}^{4}} \frac{1}{2 \pi i} \int_{\gamma} d s\left(\frac{m^{2}}{M^{2}}\right)^{1-s} \Phi(s),
\end{aligned}
$$

where

$$
\begin{aligned}
\Phi(s) & =\frac{\pi^{2} \csc ^{2}(\pi s) \Gamma(s)^{2}}{(1-s) \Gamma(2 s)} \\
& \approx \frac{2}{s^{3}}+\frac{2}{s^{2}}+\frac{6+\pi^{2}}{3} \frac{1}{s}+\mathcal{O}\left(s^{0}\right), \quad s \rightarrow 0 .
\end{aligned}
$$

The expansion after the symbol " $\approx$ " is the singular expansion of the function $\Phi(s)$. According to the converse mapping theorem [23], the expansion for $\delta a_{\mathrm{CT}}$ in powers of $m^{2} / M^{2}$ is then 


$$
\delta a_{\mathrm{CT}}=\frac{m^{4}}{f_{\pi}^{4}} \frac{m^{2}}{M^{2}}\left(\log ^{2} \frac{m^{2}}{M^{2}}-2 \log \frac{m^{2}}{M^{2}}+\frac{6+\pi^{2}}{3}\right) .
$$

The function $\Phi(s)$ has poles not only at $s=0$, but also at all the negative integers in the left half plane, but those poles lead to higher powers of $\mathrm{m}^{2} / M^{2}$. For example, near $s=-1$ the singular expansion is

$$
\begin{aligned}
\Phi(s)= & \frac{\pi^{2} \csc ^{2}(\pi s) \Gamma(s)^{2}}{(1-s) \Gamma(2 s)} \\
\approx & \frac{2}{(s+1)^{3}}-\frac{2}{(s+1)^{2}}+\frac{-15+2 \pi^{2}}{6} \frac{1}{s+1} \\
& +\mathcal{O}\left((s+1)^{0}\right),
\end{aligned}
$$

which leads to a contribution of order $\left(\mathrm{m}^{4} / f_{\pi}^{4}\right)\left(\mathrm{m}^{4} / M^{4}\right)$ times logarithms to $\delta a_{\mathrm{CT}}$.

Next, we give some details about the derivation of Eq. (3.26) from Eq. (3.25). For simplicity, we set $n=1$; the dependence on $n$ can be restored in the final result by replacing $L \rightarrow n L$. A change of variables

$$
y^{2}=m_{\sigma}^{2} L^{2}(1-\hat{x})+m_{\pi}^{2} L^{2} \hat{x} \quad \leftrightarrow \quad \hat{x}=\frac{y^{2}-m_{\sigma}^{2} L^{2}}{m_{\pi}^{2} L^{2}-m_{\sigma}^{2} L^{2}}
$$

allows us to rewrite Eq. (3.26) as

$$
\begin{aligned}
a^{(1)}= & -\frac{m_{\mu}^{2}}{16 \pi^{2}} \frac{2}{\left(m_{\sigma}^{2}-m_{\pi}^{2}\right)^{2} L^{2}} \int_{m_{\pi} L}^{m_{\sigma} L} d y\left(m_{\sigma}^{2} L^{2}-y^{2}\right) \\
& \times\left(\frac{K_{0}(y)}{y}+K_{1}(y)\left(\gamma_{E}+\log \frac{m_{\mu}^{2}\left(m_{\sigma}^{2} L^{2}-y^{2}\right)\left(y^{2}-m_{\pi}^{2} L^{2}\right)}{2\left(m_{\sigma}^{2}-m_{\pi}^{2}\right)^{2} L^{2} y}\right)\right) .
\end{aligned}
$$

Next, we will always drop terms that are manifestly suppressed by $m_{\pi}^{2} / m_{\sigma}^{2}$ or $e^{-m_{\sigma} L}$ relative to the dominant contribution. Therefore, we can replace the upper limit $m_{\sigma} L$ of the integral by $\infty$ and we may neglect $y^{2}$ in comparison with $m_{\sigma}^{2} L^{2}$ in the combinations $m_{\sigma}^{2} L^{2}-y^{2}$ appearing in Eq. (B9), because the functions $K_{0,1}(y)$ fall off exponentially like $e^{-y}$ at large $y$ and thus suppress such contributions by a factor $e^{-m_{\sigma} L}$. This simplifies Eq. (B9) to

$$
a^{(1)} \approx-\frac{1}{8 \pi^{2}} \frac{m_{\mu}^{2}}{m_{\sigma}^{2}} \int_{m_{\pi} L}^{\infty} \frac{d y}{y}\left[K_{0}(y)+y K_{1}(y)\left(\gamma_{E}+\log \frac{m_{\mu}^{2}\left(y^{2}-m_{\pi}^{2} L^{2}\right)}{2 m_{\sigma}^{2} y}\right)\right]
$$

and, using the representation

$$
K_{\nu}(y)=\frac{y^{\nu}}{2} \int_{-\infty}^{\infty} d z \frac{e^{i z}}{\left(z^{2}+y^{2}\right)^{\nu+(1 / 2)}} \quad(\nu=0,1)
$$

one may carry out the $y$ integral and obtain

$$
\begin{aligned}
a^{(1)} & \approx-\frac{1}{16 \pi^{2}} \frac{m_{\mu}^{2}}{m_{\sigma}^{2}} \int_{-\infty}^{\infty} d z \frac{e^{i z}}{\left(z^{2}+m_{\pi}^{2} L^{2}\right)^{1 / 2}}\left[\gamma_{E}+\log \frac{2 m_{\mu}^{2}\left(z^{2}+m_{\pi}^{2} L^{2}\right)}{m_{\sigma}^{2} m_{\pi} L}\right] \\
& =\frac{1}{8 \pi^{2}} \frac{m_{\mu}^{2}}{m_{\sigma}^{2}} K_{0}\left(m_{\pi} L\right) \log \frac{m_{\sigma}^{2}}{m_{\mu}^{2}} .
\end{aligned}
$$

Finally, the replacement $L \rightarrow n L$ yields the result (3.26), as promised.

[1] G. W. Bennett et al. (Muon g-2 Collaboration), Final report of the Muon E821 anomalous magnetic moment measurement at BNL, Phys. Rev. D 73, 072003 (2006).
[2] T. Aoyama et al., The anomalous magnetic moment of the muon in the standard model, arXiv:2006.04822.

[3] J. Grange et al. (Muon g-2 Collaboration), Muon (g-2) technical design report, arXiv:1501.06858. 
[4] M. Abe et al., A new approach for measuring the Muon anomalous magnetic moment and electric dipole moment, Prog. Theor. Exp. Phys. 2019, $053 \mathrm{C} 02$ (2019).

[5] A. Francis, B. Jaeger, H. B. Meyer, and H. Wittig, A new representation of the Adler function for lattice QCD, Phys. Rev. D 88, 054502 (2013).

[6] G. Gounaris and J. Sakurai, Finite Width Corrections to the Vector Meson Dominance Prediction for $\rho \rightarrow e^{+} e^{-}$, Phys. Rev. Lett. 21, 244 (1968).

[7] M. Lüscher, Signatures of unstable particles in finite volume, Nucl. Phys. B364, 237 (1991); L. Lellouch and M. Lüscher, Weak transition matrix elements from finite volume correlation functions, Commun. Math. Phys. 219, 31 (2001).

[8] C. Aubin, T. Blum, P. Chau, M. Golterman, S. Peris, and C. Tu, Finite-volume effects in the muon anomalous magnetic moment on the lattice, Phys. Rev. D 93, 054508 (2016).

[9] J. Bijnens and J. Relefors, Vector two-point functions in finite volume using partially quenched chiral perturbation theory at two loops, J. High Energy Phys. 12 (2017) 114.

[10] C. Aubin, T. Blum, C. Tu, M. Golterman, C. Jung, and S. Peris, Light quark vacuum polarization at the physical point and contribution to the muon $g-2$, Phys. Rev. D 101, 014503 (2020).

[11] S. Borsanyi, Z. Fodor, J. Guenther, C. Hoelbling, S. Katz, L. Lellouch, T. Lippert, K. Miura, L. Parato, K. Szabo, F. Stokes, B. Toth, C. Torok, and L. Varnhorst, Leading-order hadronic vacuum polarization contribution to the muon magnetic moment from lattice QCD, arXiv:2002.12347.

[12] J. Gasser and H. Leutwyler, Spontaneously broken symmetries: Effective Lagrangians at finite volume, Nucl. Phys. B307, 763 (1988).

[13] B. Chakraborty, C. Davies, P. de Oliviera, J. Koponen, G. Lepage, and R. Van de Water, The hadronic vacuum polarization contribution to $a_{\mu}$ from full lattice QCD, Phys. Rev. D 96, 034516 (2017).

[14] M. T. Hansen and A. Patella, Finite-Volume Effects in $(g-2)_{\mu}^{\mathrm{HVP}, \mathrm{LO}}$, Phys. Rev. Lett. 123, 172001 (2019).
[15] M. T. Hansen and A. Patella, Finite-volume and thermal effects in the leading-HVP contribution to muonic $(g-2)$, J. High Energy Phys. 10 (2020) 029.

[16] M. Lüscher, Volume dependence of the energy spectrum in massive quantum field theories. 1. Stable particle states, Commun. Math. Phys. 104, 177 (1986).

[17] E. Shintani, and Y. Kuramashi (PACS Collaboration), Hadronic vacuum polarization contribution to the muon $g-2$ with $2+1$ flavor lattice QCD on a larger than $(10 \mathrm{fm})^{4}$ lattice at the physical point, Phys. Rev. D 100, 034517 (2019).

[18] T. Blum, Lattice Calculation of the Lowest Order Hadronic Contribution to the Muon Anomalous Magnetic Moment, Phys. Rev. Lett. 91, 052001 (2003).

[19] B.E. Lautrup, A. Peterman, and E. de Rafael, Recent developments in the comparison between theory and experiments in quantum electrodynamics, Phys. Rep. 3, 193 (1972).

[20] D. Bernecker and H. B. Meyer, Vector correlators in Lattice QCD: Methods and applications, Eur. Phys. J. A 47, 148 (2011).

[21] J. Bijnens, G. Colangelo, and G. Ecker, The mesonic chiral Lagrangian of order $p^{6}$, J. High Energy Phys. 02 (1999) 020.

[22] N. Asmussen, J. Green, H. B. Meyer, and A. Nyffeler, Position-space approach to hadronic light-by-light scattering in the muon $g-2$ on the lattice, Proc. Sci., LATTICE2016 (2016) 164 [arXiv:1609.08454]; T. Blum, N. Christ, M. Hayakawa, T. Izubuchi, L. Jin, C. Jung, and C. Lehner, Using infinite volume, continuum QED and lattice QCD for the hadronic light-by-light contribution to the muon anomalous magnetic moment, Phys. Rev. D 96, 034515 (2017); N. Asmussen, A. Gérardin, H. B. Meyer, and A. Nyffeler, Exploratory studies for the position-space approach to hadronic light-by-light scattering in the muon $g-2$, EPJ Web Conf. 175, 06023 (2018).

[23] P. Flajolet, X. Gourdon, and P. Dumas, Mellin transforms and asymptotic harmonic sums, Theor. Comput. Sci. 144, 3 (1995). 